MATHEMATICS OF COMPUTATION

Volume 81, Number 278, April 2012, Pages 623-642

S $0025-5718(2011) 02544-5$

Article electronically published on December 13, 2011

\title{
CONVERGENCE AND OPTIMALITY OF ADAPTIVE EDGE FINITE ELEMENT METHODS FOR TIME-HARMONIC MAXWELL EQUATIONS
}

\author{
LIUQIANG ZHONG, LONG CHEN, SHI SHU, GABRIEL WITTUM, AND JINCHAO XU
}

\begin{abstract}
We consider a standard Adaptive Edge Finite Element Method (AEFEM) based on arbitrary order Nédélec edge elements, for three-dimensional indefinite time-harmonic Maxwell equations. We prove that the AEFEM gives a contraction for the sum of the energy error and the scaled error estimator, between two consecutive adaptive loops provided the initial mesh is fine enough. Using the geometric decay, we show that the AEFEM yields the best possible decay rate of the error plus oscillation in terms of the number of degrees of freedom. The main technical contribution of the paper is the establishment of a quasi-orthogonality and a localized a posteriori error estimator.
\end{abstract}

\section{INTRODUCTION}

Let $\Omega$ be a bounded and Lipschitz domain in $\mathbb{R}^{3}$ with a connected boundary $\partial \Omega$ and unit outward normal $\boldsymbol{n}_{\partial \Omega}$. We consider the following classical time-harmonic Maxwell equations:

$$
\begin{array}{rll}
\nabla \times(\nabla \times \boldsymbol{u})-\omega^{2} \boldsymbol{u} & =\boldsymbol{g} & \text { in } \Omega, \\
\boldsymbol{u} \times \boldsymbol{n}_{\partial \Omega} & =0 & \text { on } \partial \Omega,
\end{array}
$$

where $\boldsymbol{u}$ is the electric field, the real and positive constant $\omega$ is a wave number of the electromagnetic wave, and $\boldsymbol{g} \in \boldsymbol{L}^{2}(\Omega)$ is a given function related to the imposed current sources. The boundary condition (1.2) is chosen for simplicity of exposition. Our results can easily be generalized to other types of boundary conditions. In order to have a well-posed problem, we assume that $\omega^{2}$ is not an eigenvalue of the differential operator $\mathcal{L}:=\nabla \times(\nabla \times)$. Furthermore, we assume that $\nabla \cdot \boldsymbol{g} \in L^{2}(\Omega)$; this represents the charge density in electromagnetics (see 29, Section 1.2).

Received by the editor June 3, 2010.

2010 Mathematics Subject Classification. Primary 65F10, 65N30; Secondary 65N12, 78A25.

The first author was supported in part by the National Natural Science Foundation of China (Grant No. 11026091).

The second author was supported by NSF Grant DMS-0811272 and in part by 2010-2011 UC Irvine CORCL.

The third author was supported in part by NSFC Key Project 11031006, Hunan Provincial Natural Science Foundation of China (Grant No. 10JJ7001), and the Aid program for Science and Technology Innovative Research Team in Higher Educational Institutions of Hunan Province of China.

The fourth author was supported in part by the German ministry of Economics and technology, BMWi, and the GRS (Gesellschaft für Reaktorsicherheit).

The last author was supported in part by NSF Grant DMS-0915153, DMS-0749202 and the Alexander von Humboldt Research Award for Senior US Scientist. 
Finite element methods based on Nédélec edge elements [33, 34] are one of the most popular choices for the numerical computation of Maxwell equations. In many applications, the solution of (1.1) and (1.2) presents strong singularities and an adaptive edge finite element method (AEFEM) is needed to capture the singularity in an efficient way.

In this paper, we are interested in the theoretical understanding of the adaptive edge finite element methods for time-harmonic Maxwell equations. We shall prove the convergence and optimality of the following standard adaptive procedure using edge elements:

$$
\text { SOLVE } \rightarrow \text { ESTIMATE } \rightarrow \text { MARK } \rightarrow \text { REFINE. }
$$

(The precise definition of the algorithm can be found in §2). In recent years, mathematicians have started to prove the convergence and optimal complexity of the adaptive procedure in the form of (1.3) [15, 31, 32, 6, 40, 8. We refer to [35] for an introduction to the theory of adaptive finite element methods.

For Maxwell equations, the convergence analysis of adaptive procedures is established for the two- and three-dimensional eddy currents equations in [7] and 22, respectively. In these works the convergence analysis relied on the so-called interior node property and an extra marking for oscillation which both seem to be not necessary in practice.

In this paper, we shall follow the state-of-the-art convergence theory [8] to prove the convergence without interior node property and extra marking for oscillation, and, more importantly, to establish the quasi-optimal convergent rate of the AEFEM. Technically speaking, the main contribution of this paper is to establish two important ingredients used in the framework developed in [8, namely quasiorthogonality and a localized upper bound. We stress that the extension of the general convergence theory to the time-harmonic Maxwell equation is not straightforward. Both the quasi-orthogonality and localized upper bound require highly non-trivial techniques.

More precisely, this paper's contributions include:

(1) an analysis of the indefinite time-harmonic Maxwell equations using the approach for non-symmetric elliptic equations in [44, 25. We emphasize that in our case, the $L^{2}$ estimate is much more difficult than that of elliptic equations since the standard duality approach does not work. We adapt the technique from Gopalakrishnan and Pasciak [16].

(2) a derivation of a quasi-optimal rate of convergence for the AEFEM. This result seems to be the first result of this type for Maxwell equations. The crucial technique is to prove a localized upper bound. To this end, we construct a stable and local projection operator between two consecutive finite element spaces and use a localized regular decomposition developed by Schöberl 38.

There are still some interesting questions that need to be further investigated. For example, the rate of convergence in this paper is optimal restricted to isotropic refinement (bisection grids). Anisotropic refinement might further improve the convergence for some special cases [14]. However, it is difficult to realize a posteriori, i.e., without knowing the asymptotic of the singularity. It should also be remarked that the scheme is not uniform with respect to the wave number. In fact, we need 
to assume that the initial grid is sufficiently fine, which seems to be necessary for finite element approximations [27, 48, 16].

To avoid the repeated use of generic but unspecified constants, following [43], we shall use the following shorthand notation: $x \lesssim y$ means $x \leq C y, x \gtrsim y$ means $x \geq c y$, and $x \bar{\sim} y$ means $c x \leq y \leq C y$, where $c$ and $C$ are generic positive constants independent of the variables that appear in the inequalities and especially the mesh parameters. The notation $C_{i}$, with subscript, denotes specific and important constants.

The rest of this article is organized as follows. We describe the variational formulation of the model problem and discuss each procedure of (1.3) in $\S 2$ in detail. We prove the convergence and optimal complexity of the AEFEM in Sections 3 and 4 , respectively.

\section{An ADAPTIVE EDGE Finite ELEMENT METHOD}

In this section, we shall introduce the variational formulation of the model problem and present an adaptive edge finite element method.

2.1. Variational formulation. For any open set $G \subset \mathbb{R}^{3}, L^{2}(G)$ or $\boldsymbol{L}^{2}(G)$ stands for the Hilbert space of square integrable functions or vector fields, respectively, on $G$ with inner product $(\cdot, \cdot)_{G}$, and $H^{1}(G):=\left\{v \in L^{2}(G): \nabla v \in \boldsymbol{L}^{2}(G)\right\}$. We also define the spaces

$$
\begin{aligned}
\boldsymbol{H}(\mathbf{c u r l} ; G) & =\left\{\boldsymbol{v} \in \boldsymbol{L}^{2}(G) \mid \nabla \times \boldsymbol{v} \in \boldsymbol{L}^{2}(G)\right\}, \\
\boldsymbol{H}(\operatorname{div} ; G) & =\left\{\boldsymbol{v} \in \boldsymbol{L}^{2}(G) \mid \nabla \cdot \boldsymbol{v} \in L^{2}(G)\right\},
\end{aligned}
$$

equipped with norms

$$
\begin{aligned}
\|\boldsymbol{v}\|_{\text {curl } ; G} & =\left(\|\boldsymbol{v}\|_{0 ; G}^{2}+\|\nabla \times \boldsymbol{v}\|_{0 ; G}^{2}\right)^{1 / 2}, \quad \text { for all } \boldsymbol{v} \in \boldsymbol{H}(\mathbf{c u r l} ; G), \\
\|\boldsymbol{v}\|_{\operatorname{div} ; G} & =\left(\|\boldsymbol{v}\|_{0 ; G}^{2}+\|\nabla \cdot \boldsymbol{v}\|_{0 ; G}^{2}\right)^{1 / 2}, \quad \text { for all } \boldsymbol{v} \in \boldsymbol{H}(\operatorname{div} ; G),
\end{aligned}
$$

respectively, where $\|\cdot\|_{0 ; G}:=(\cdot, \cdot)_{G}^{1 / 2}$ denotes the norm of space $\boldsymbol{L}^{2}(G)$ or $L^{2}(G)$. In particular, we define $H_{0}^{1}(G):=\left\{u \in H^{1}(G),\left.u\right|_{\partial G}=0\right\}$ and $\boldsymbol{H}_{0}(\mathbf{c u r l} ; G)=\{\boldsymbol{u} \in$ $\boldsymbol{H}(\operatorname{curl} ; G), \boldsymbol{n}_{\partial G} \times \boldsymbol{u}=\mathbf{0}$ on $\partial G$ in the trace sense $\}$, where $\boldsymbol{n}_{\partial G}$ denotes the unit outward normal of the boundary $\partial G$ of domain $G$. For simplicity of notation, when $G=\Omega$, it will be omitted in the subscript.

The variational formulation of equations (1.1) and (1.2) is: Find $\boldsymbol{u} \in \boldsymbol{H}_{0}(\mathbf{c u r l} ; \Omega)$, such that

$$
\hat{a}(\boldsymbol{u}, \boldsymbol{v})=(\boldsymbol{g}, \boldsymbol{v}), \quad \text { for all } \boldsymbol{v} \in \boldsymbol{H}_{0}(\operatorname{curl} ; \Omega),
$$

where the bilinear form

$$
\hat{a}(\boldsymbol{u}, \boldsymbol{v}):=(\nabla \times \boldsymbol{u}, \nabla \times \boldsymbol{v})-\omega^{2}(\boldsymbol{u}, \boldsymbol{v}) .
$$

We assume $\omega^{2}$ is not an eigenvalue of the differential operator $\mathcal{L}:=\nabla \times(\nabla \times)$. Then the well-poseness of the variational problem (2.1) follows from the Fredholm alternative theorem; cf., Chapter 4 of 29 . In this case, there exists a constant 
$\alpha_{0}>0$ depending only on $\Omega$ and the wave number $\omega$ such that the following infsup conditions hold:

$$
\begin{aligned}
& \inf _{\boldsymbol{v} \in \boldsymbol{H}_{0}(\mathbf{c u r l} ; \Omega)} \sup _{\boldsymbol{w} \in \boldsymbol{H}_{0}(\operatorname{curl} ; \Omega)} \frac{\hat{a}(\boldsymbol{v}, \boldsymbol{w})}{\|\boldsymbol{v}\|_{\text {curl }}\|\boldsymbol{w}\|_{\text {curl }}} \\
& \quad=\inf _{\boldsymbol{w} \in \boldsymbol{H}_{0}(\operatorname{curl} ; \Omega)} \sup _{\boldsymbol{v} \in \boldsymbol{H}_{0}(\operatorname{curl} ; \Omega)} \frac{\hat{a}(\boldsymbol{v}, \boldsymbol{w})}{\|\boldsymbol{v}\|_{\text {curl }}\|\boldsymbol{w}\|_{\text {curl }}}=\alpha_{0}>0 .
\end{aligned}
$$

2.2. Edge finite element methods. Nédélec 33, 34 type $\boldsymbol{H}$ (curl)-conforming finite elements are the natural choice for discretization of the variational problem (2.1). For each positive integer $l, \mathcal{P}_{l}$ denotes the standard space of polynomials of total degree less than or equal to $l$, and $\tilde{\mathcal{P}}_{l}$ denotes the space of homogeneous polynomials of degree $l$. For any given conforming triangulation $\mathcal{T}$, the $l^{\text {th }}$-order element of the first family and second family of Nédélec elements is defined by

$$
\begin{aligned}
& \mathbb{V}^{l, 1}(\mathcal{T}):=\left\{\boldsymbol{v}_{h}^{l, 1} \in \boldsymbol{H}_{0}(\operatorname{curl} ; \Omega)\left|\boldsymbol{v}_{h}^{l, 1}\right|_{K} \in \mathcal{R}_{l} \text { for all } K \in \mathcal{T}\right\} \\
& \mathbb{V}^{l, 2}(\mathcal{T}):=\left\{\boldsymbol{v}_{h}^{l, 2} \in \boldsymbol{H}_{0}(\operatorname{curl} ; \Omega)\left|\boldsymbol{v}_{h}^{l, 2}\right|_{K} \in\left(\mathcal{P}_{l}\right)^{3} \text { for all } K \in \mathcal{T}\right\}
\end{aligned}
$$

where $\mathcal{R}_{l}:=\left(\mathcal{P}_{l-1}\right)^{3} \oplus\left\{\boldsymbol{p} \in\left(\tilde{P}_{l}\right)^{3} \mid \boldsymbol{p}(\boldsymbol{x}) \cdot \boldsymbol{x}=\mathbf{0}\right\}$.

To save notation, we use $\mathbb{V}(\mathcal{T})$ for both first- and second-type Nédélec element spaces. The lowest-order element of the first family $\mathbb{V}^{1,1}(\mathcal{T})$ is the simplest one, and $\mathbb{V}^{1,1}(\mathcal{T}) \subseteq \mathbb{V}(\mathcal{T})$ is always true.

The edge finite element methods for solving (2.1) is: Find $\boldsymbol{u}_{\mathcal{T}} \in \mathbb{V}(\mathcal{T})$, such that

$$
\hat{a}\left(\boldsymbol{u}_{\mathcal{T}}, \boldsymbol{v}_{\mathcal{T}}\right)=\left(\boldsymbol{g}, \boldsymbol{v}_{\mathcal{T}}\right), \quad \text { for all } \boldsymbol{v}_{\mathcal{T}} \in \mathbb{V}(\mathcal{T})
$$

The existence of the finite element solution of (2.3) has been proved provided that the mesh size $h_{\mathcal{T}}:=\max _{K \in \mathcal{T}} \operatorname{diam}(K)$ is sufficiently small; see Monk [27, Hiptmair [19], and Zhong, Shu, Wittum and Xu [48. We shall always assume that the initial mesh size $h_{0}:=h_{\mathcal{T}_{0}}$ is sufficiently small, such that (2.3) is well-posed. Namely, there exists a constant $\alpha_{1}>0$, such that for all $\mathcal{T} \in \mathscr{C}\left(\mathcal{T}_{0}\right)$, where $\mathscr{C}\left(\mathcal{T}_{0}\right)$ is a class of conforming triangulations refined from $\mathcal{T}_{0}$ defined in 2.3 .4 the following inf-sup conditions hold:

$$
\inf _{\boldsymbol{v}_{\mathcal{T}} \in \mathbb{V}(\mathcal{T})} \sup _{\boldsymbol{w}_{\mathcal{T} \in \mathbb{V}(\mathcal{T})}} \frac{\hat{a}\left(\boldsymbol{v}_{\mathcal{T}}, \boldsymbol{w}_{\mathcal{T}}\right)}{\left\|\boldsymbol{v}_{\mathcal{T}}\right\|_{\text {curl }}\left\|\boldsymbol{w}_{\mathcal{T}}\right\|_{\text {curl }}}=\inf _{\boldsymbol{w}_{\mathcal{T}} \in \mathbb{V}(\mathcal{T})} \sup _{\boldsymbol{v}_{\mathcal{T}} \in \mathbb{V}(\mathcal{T})} \frac{\hat{a}\left(\boldsymbol{v}_{\mathcal{T}}, \boldsymbol{w}_{\mathcal{T}}\right)}{\left\|\boldsymbol{v}_{\mathcal{T}}\right\|_{\text {curl }}\left\|\boldsymbol{w}_{\mathcal{T}}\right\|_{\text {curl }}} \geq \alpha_{1}
$$

2.3. An adaptive edge finite element method. The solution of (2.1) may contain strong singularities caused by various sources, such as physical domains with non-trivial geometries, discontinuous material coefficients, and non-smooth source terms [12, 13. We present the following algorithm to resolve the singularity. 


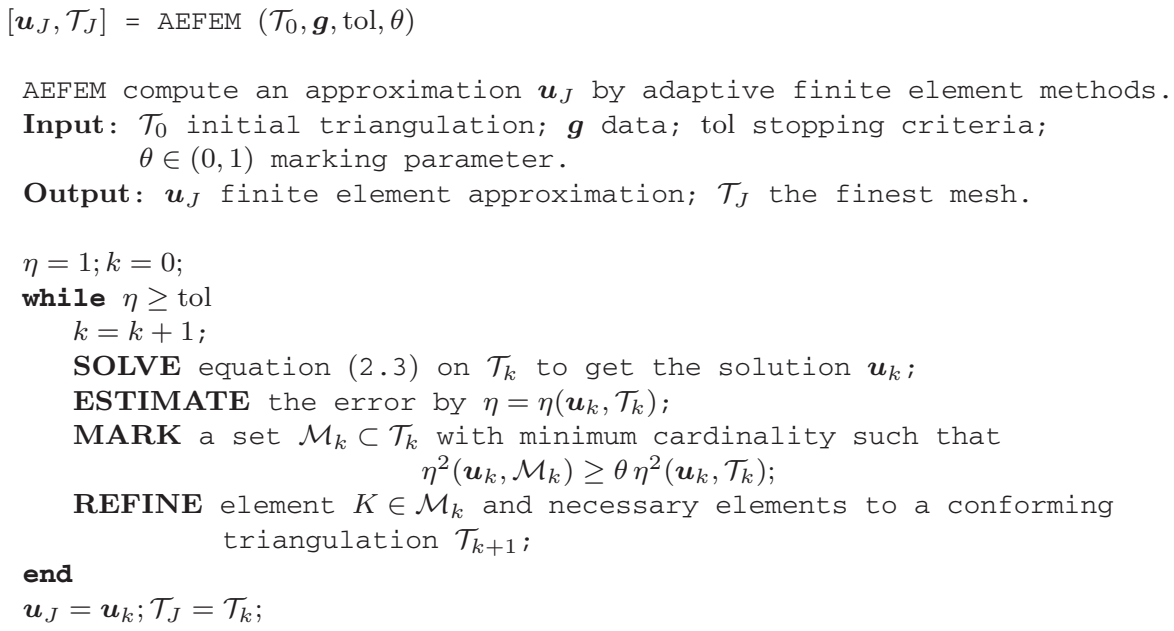

The goal of this paper is to prove that the algorithm AEFEM will terminate in finite steps for a given tolerance and produce a quasi-optimal approximation $\boldsymbol{u}_{J}$. Our algorithm is similar to that for second-order elliptic PDEs in 8. It is the simplest adaptive algorithm in the sense that no marking for oscillation and no interior node property should be enforced in the mark and refine procedure.

In the following sections, we shall discuss each step in detail.

2.3.1. Procedure SOLVE. Given a function $\boldsymbol{g} \in \boldsymbol{L}^{\mathbf{2}}(\Omega)$ and a mesh $\mathcal{T}$, we suppose that the procedure $\boldsymbol{u}_{\mathcal{T}}=\operatorname{SOLVE}(\mathcal{T}, \boldsymbol{g})$ outputs the exact discrete solution $\boldsymbol{u}_{\mathcal{T}} \in$ $\mathbb{V}(\mathcal{T})$ solving (2.3). Here, we assume that the solutions of the finite dimensional problems can be solved accurately and efficiently. Examples of such optimal solvers include multigrid methods [18, 2, 17, 10, 20, domain decomposition preconditioners 1. 16, and two-grid methods 47. We note that most of the above studies focus on quasi-uniform grids. Multigrid methods for the $\boldsymbol{H}$ (curl) problem on adaptive grids can be found in 21, 9, 45].

2.3.2. Procedure ESTIMATE. For the $\boldsymbol{H}$ (curl)-system, efficient and reliable a posteriori error estimators have been extensively developed and analyzed in 4, 5, 10, 28, 38. Here, we shall use a residual-type a posteriori error estimator similar to that in 38. Given a conforming triangulation $\mathcal{T}$, let $\mathcal{F}(\mathcal{T})$ denote the set of the interior faces of $\mathcal{T}$ with a fixed orientation for each face. For a face $f \in \mathcal{F}(\mathcal{T})$ shared by two elements $K_{1}$ and $K_{2}$, i.e., $\partial K_{1} \cap \partial K_{2}=f$ with the orientation of $f$ being consistent with that of $K_{1}$, we define the interelement jumps of a scalar function $w$ across $f$ as

$$
\llbracket w \rrbracket=\left.w\right|_{K_{1}}-\left.w\right|_{K_{2}} .
$$

For $K \in \mathcal{T}, f \in \mathcal{F}(\mathcal{T})$ and $\boldsymbol{v}_{\mathcal{T}} \in \mathbb{V}(\mathcal{T})$, we define the following elementwise residuals and facewise jump residuals associated with interior faces as

$$
\begin{aligned}
\left.R_{1}\left(\boldsymbol{v}_{\mathcal{T}}\right)\right|_{K} & :=\left.\left(\boldsymbol{g}-\nabla \times\left(\nabla \times \boldsymbol{v}_{\mathcal{T}}\right)+\omega^{2} \boldsymbol{v}_{\mathcal{T}}\right)\right|_{K}, \\
\left.J_{1}\left(\boldsymbol{v}_{\mathcal{T}}\right)\right|_{f} & :=\llbracket\left(\nabla \times \boldsymbol{v}_{\mathcal{T}}\right) \times \boldsymbol{n}_{f} \rrbracket, \\
\left.R_{2}\left(\boldsymbol{v}_{\mathcal{T}}\right)\right|_{K} & :=\nabla \cdot\left(\left.\boldsymbol{g}\right|_{K}+\left.\omega^{2} \boldsymbol{v}_{\mathcal{T}}\right|_{K}\right), \\
\left.J_{2}\left(\boldsymbol{v}_{\mathcal{T}}\right)\right|_{f} & :=\llbracket\left(\boldsymbol{g}+\omega^{2} \boldsymbol{v}_{\mathcal{T}}\right) \cdot \boldsymbol{n}_{f} \rrbracket .
\end{aligned}
$$


The error indicator for $\boldsymbol{v}_{\mathcal{T}} \in \mathbb{V}(\mathcal{T})$ on $K \in \mathcal{T}$ is given by

$$
\begin{aligned}
\eta_{\mathcal{T}}^{2}\left(\boldsymbol{v}_{\mathcal{T}}, K\right):= & h_{K}^{2}\left(\left\|R_{1}\left(\boldsymbol{v}_{\mathcal{T}}\right)\right\|_{0 ; K}^{2}+\left\|R_{2}\left(\boldsymbol{v}_{\mathcal{T}}\right)\right\|_{0 ; K}^{2}\right) \\
& +\sum_{f \in K \cap \mathcal{F}(\mathcal{T})} h_{K}\left(\left\|J_{1}\left(\boldsymbol{v}_{\mathcal{T}}\right)\right\|_{0 ; f}^{2}+\left\|J_{2}\left(\boldsymbol{v}_{\mathcal{T}}\right)\right\|_{0 ; f}^{2}\right),
\end{aligned}
$$

where $|K|$ is the volume of $K$ and $h_{K}=|K|^{1 / 3}$ measures the size of the element $K$.

For any subset $\mathcal{M} \subseteq \mathcal{T}$, we define

$$
\eta_{\mathcal{T}}^{2}\left(\boldsymbol{v}_{\mathcal{T}}, \mathcal{M}\right)=\sum_{K \in \mathcal{M}} \eta_{\mathcal{T}}^{2}\left(\boldsymbol{v}_{\mathcal{T}}, K\right)
$$

When $\mathcal{M}=\mathcal{T}$, we simplify the notation as $\eta\left(\boldsymbol{v}_{\mathcal{T}}, \mathcal{T}\right)$.

We assume that, given a triangulation $\mathcal{T}$ and the corresponding discrete solution $\boldsymbol{u}_{\mathcal{T}} \in \mathbb{V}(\mathcal{T})$ of (2.3), the procedure ESTIMATE outputs the indicators $\eta_{\mathcal{T}}\left(\boldsymbol{v}_{\mathcal{T}}, K\right)$ for all $K \in \mathcal{T}$.

2.3.3. Procedure $M A R K$. In the selection of elements, we rely on the Dörfler marking, also known as bulk criterion [15. Given a triangulation $\mathcal{T}$, a set of indicators $\left\{\eta_{\mathcal{T}}\left(\boldsymbol{u}_{\mathcal{T}}, K\right)\right\}_{K \in \mathcal{T}}$, and a marking parameter $\theta \in(0,1)$, we suppose that the procedure MARK outputs a subset of marked elements $\mathcal{M} \subset \mathcal{T}$ with minimal cardinality, such that

$$
\eta_{\mathcal{T}}^{2}\left(\boldsymbol{u}_{\mathcal{T}}, \mathcal{M}\right) \geq \theta \eta^{2}\left(\boldsymbol{u}_{\mathcal{T}}, \mathcal{T}\right)
$$

2.3.4. Procedure REFINE. We use bisection methods for the local mesh refinement. In short, bisection methods will divide one simplex into two simplicies of equal size in a proper way such that the meshes obtained by bisection are shape regular.

Starting from an initial triangulation $\mathcal{T}_{0}$, we denote by

$$
\mathscr{C}\left(\mathcal{T}_{0}\right)=\left\{\mathcal{T}: \mathcal{T} \text { is conforming and refined from } \mathcal{T}_{0}\right\},
$$

and $\mathcal{T}_{1} \leq \mathcal{T}_{2}$ if $\mathcal{T}_{2}$ is a refinement of $\mathcal{T}_{1}$.

For any $\mathcal{T}_{k} \in \mathscr{C}\left(\mathcal{T}_{0}\right)$ and a subset $\mathcal{M}_{k} \subset \mathcal{T}_{k}$ of marked elements, we suppose that procedure REFINE outputs a conforming triangulation $\mathcal{T}_{k+1} \in \mathscr{C}\left(\mathcal{T}_{0}\right)$, i.e.,

$$
\mathcal{T}_{k+1}=\operatorname{REFINE}\left(\mathcal{T}_{k}, \mathcal{M}_{k}\right) .
$$

To generate $\mathcal{T}_{k+1}$, we first subdivide the marked elements in $\mathcal{M}_{k}$ to get new triangulation $\mathcal{T}_{k+1}^{\prime}$. In general, $\mathcal{T}_{k+1}^{\prime}$ might have hanging nodes; therefore, we have to refine additional elements in $\mathcal{T}_{k} \backslash \mathcal{M}_{k}$ to obtain a conforming triangulation $\mathcal{T}_{k+1}$.

Throughout this paper, we shall impose two conditions on the local refinement:

(B1) $\mathscr{C}\left(\mathcal{T}_{0}\right)$ is shape regular;

(B2) There exists a constant $C_{0}$ depending on the shape regularity of $\mathcal{T}_{0}$, such that

$$
\# \mathcal{T}_{k+1}-\# \mathcal{T}_{0} \leq C_{0} \sum_{j=0}^{k} \# \mathcal{M}_{j}
$$

Result (2.5) for the newest vertex bisection in 2-D was first proved by Binev, Dahmen, and DeVore [6] based on an initial labeling of Mitchell [26]. It was generalized to high dimensions by Stevenson [41] using a Kossaczkỳ-type initial labeling 24 . 


\section{Convergence of the AEFEM}

In this section, we prove that the sum of the energy error and the scaled error estimator, between two consecutive adaptive loops, is a contraction. The difficulty is to establish a quasi-orthogonality property for the indefinite time-harmonic Maxwell equations.

3.1. Quasi-orthogonality. We define two auxiliary bilinear forms:

$$
\begin{aligned}
a(\boldsymbol{v}, \boldsymbol{w}) & =(\nabla \times \boldsymbol{v}, \nabla \times \boldsymbol{w})+(\boldsymbol{v}, \boldsymbol{w}), \\
N(\boldsymbol{v}, \boldsymbol{w}) & =-\left(\omega^{2}+1\right)(\boldsymbol{v}, \boldsymbol{w}) .
\end{aligned}
$$

By definition, $\hat{a}(\boldsymbol{v}, \boldsymbol{w})=a(\boldsymbol{v}, \boldsymbol{w})+N(\boldsymbol{v}, \boldsymbol{w})$. The bilinear form $a(\cdot, \cdot)$ forms the standard inner product of the $\boldsymbol{H}(\mathbf{c u r l} ; \Omega)$ space, and $N(\cdot, \cdot)$ is a lower-order part in view of the differential operator.

Lemma 3.1. For $\mathcal{T}, \mathcal{T}_{*} \in \mathscr{C}\left(\mathcal{T}_{0}\right)$ with $\mathcal{T} \leq \mathcal{T}_{*}$, let $\boldsymbol{u}_{\mathcal{T}} \in \mathbb{V}(\mathcal{T})$ and $\boldsymbol{u}_{\mathcal{T}_{*}} \in \mathbb{V}\left(\mathcal{T}_{*}\right)$ be the discrete solutions of (2.3). Then for any $\delta_{0}>0$, there exists an $h\left(\delta_{0}\right)$ depending on the parameter $\omega$, the domain $\Omega$ and $\delta_{0}$, such that, if $h_{\mathcal{T}} \leq h\left(\delta_{0}\right)$, we have

$$
N\left(\boldsymbol{u}-\boldsymbol{u}_{\mathcal{T}_{*}}, \boldsymbol{u}_{\mathcal{T}_{*}}-\boldsymbol{u}_{\mathcal{T}}\right) \leq \delta_{0}\left\|\boldsymbol{u}-\boldsymbol{u}_{\mathcal{T}_{*}}\right\|_{\text {curl }}\left\|\boldsymbol{u}_{\mathcal{T}_{*}}-\boldsymbol{u}_{\mathcal{T}}\right\|_{\text {curl }}
$$

The proof of the above lemma is rather technical and will be postponed to the next subsection. We shall use it to derive a quasi-orthogonality result.

Theorem 3.2. For $\mathcal{T}, \mathcal{T}_{*} \in \mathscr{C}\left(\mathcal{T}_{0}\right)$ with $\mathcal{T} \leq \mathcal{T}_{*}$, let $\boldsymbol{u}_{\mathcal{T}} \in \mathbb{V}(\mathcal{T})$ and $\boldsymbol{u}_{\mathcal{T}_{*}} \in \mathbb{V}\left(\mathcal{T}_{*}\right)$ be the discrete solutions of (2.3). Then for any $\delta_{0}>0$, there exists a constant $h\left(\delta_{0}\right)$ depending on the parameter $\omega$, the domain $\Omega$ and $\delta_{0}$, such that, if $h_{\mathcal{T}} \leq h\left(\delta_{0}\right)$, we have

$$
\begin{array}{r}
\left\|\boldsymbol{u}-\boldsymbol{u}_{\mathcal{T}}\right\|_{\text {curl }}^{2} \leq\left(1+\delta_{0}\right)\left(\left\|\boldsymbol{u}-\boldsymbol{u}_{\mathcal{T}_{*}}\right\|_{\text {curl }}^{2}+\left\|\boldsymbol{u}_{\mathcal{T}_{*}}-\boldsymbol{u}_{\mathcal{T}}\right\|_{\text {curl }}^{2}\right) \\
\left\|\boldsymbol{u}-\boldsymbol{u}_{\mathcal{T}_{*}}\right\|_{\text {curl }}^{2} \leq \frac{1}{1-\delta_{0}}\left\|\boldsymbol{u}-\boldsymbol{u}_{\mathcal{T}}\right\|_{\text {curl }}^{2}-\left\|\boldsymbol{u}_{\mathcal{T}_{*}}-\boldsymbol{u}_{\mathcal{T}}\right\|_{\text {curl }}^{2}
\end{array}
$$

Proof. Using the definitions of $\hat{a}(\cdot, \cdot), a(\cdot, \cdot)$ and $N(\cdot, \cdot)$, we have

$$
\begin{aligned}
\left\|\boldsymbol{u}-\boldsymbol{u}_{\mathcal{T}}\right\|_{\text {curl }}^{2}= & a\left(\boldsymbol{u}-\boldsymbol{u}_{\mathcal{T}}, \boldsymbol{u}-\boldsymbol{u}_{\mathcal{T}}\right) \\
= & a\left(\boldsymbol{u}-\boldsymbol{u}_{\mathcal{T}_{*}}, \boldsymbol{u}-\boldsymbol{u}_{\mathcal{T}_{*}}\right)+a\left(\boldsymbol{u}_{\mathcal{T}_{*}}-\boldsymbol{u}_{\mathcal{T}}, \boldsymbol{u}_{\mathcal{T}_{*}}-\boldsymbol{u}_{\mathcal{T}}\right) \\
& +2 a\left(\boldsymbol{u}-\boldsymbol{u}_{\mathcal{T}_{*}}, \boldsymbol{u}_{\mathcal{T}_{*}}-\boldsymbol{u}_{\mathcal{T}}\right) \\
= & \left\|\boldsymbol{u}-\boldsymbol{u}_{\mathcal{T}_{*}}\right\|_{\text {curl }}^{2}+\left\|\boldsymbol{u}_{\mathcal{T}_{*}}-\boldsymbol{u}_{\mathcal{T}}\right\|_{\text {curl }}^{2} \\
& -2 N\left(\boldsymbol{u}-\boldsymbol{u}_{\mathcal{T}_{*}}, \boldsymbol{u}_{\mathcal{T}_{*}}-\boldsymbol{u}_{\mathcal{T}}\right) .
\end{aligned}
$$

In the last step, we apply the Galerkin orthogonality $\hat{a}\left(\boldsymbol{u}-\boldsymbol{u}_{\mathcal{T}_{*}}, \boldsymbol{u}_{\mathcal{T}_{*}}-\boldsymbol{u}_{\mathcal{T}}\right)=0$.

Applying (3.1) and the Cauchy-Schwarz inequality in (3.4), we obtain (3.2). The inequality (3.3) can be proved similarly.

3.2. Estimate for the lower-order bilinear form. For any $s>0$, we define the Sobolev space

$$
\boldsymbol{H}^{s}(\operatorname{curl} ; \Omega)=\left\{\boldsymbol{u} \in\left(H^{s}(\Omega)\right)^{3} \mid \nabla \times \boldsymbol{u} \in\left(H^{s}(\Omega)\right)^{3}\right\},
$$

equipped with the norm

$$
\|\boldsymbol{v}\|_{\boldsymbol{H}^{s}(\mathbf{c u r l} ; \Omega)}=\left(\|\boldsymbol{v}\|_{H^{s}(\Omega)}^{2}+\|\nabla \times \boldsymbol{v}\|_{H^{s}(\Omega)}^{2}\right)^{1 / 2} .
$$

Given a triangulation $\mathcal{T} \in \mathscr{C}\left(\mathcal{T}_{0}\right)$, then for any $K \in \mathcal{T}$, the degrees of freedom for the edge finite space $\mathbb{V}(\mathcal{T})$ are of three types associated with edges $e$, faces $f$, and 
$K$ itself; see 33 and 34 for details. Using the above degrees of freedom, one can define the standard interpolation $\Pi_{\mathcal{T}}$ onto the finite element space $\mathbb{V}(\mathcal{T})$ 33, 34, 29, This interpolation is also termed canonical edge interpolation $\Pi_{\mathcal{T}_{*}}^{\text {curl }}$ for the lowestorder element of the first family, since the degrees of freedom are only associated with edges of the mesh. The next lemma states the interpolation error estimate.

Lemma 3.3 (Thm. 5.41 in [29]). If $\boldsymbol{v} \in \boldsymbol{H}^{\sigma}(\mathbf{c u r l} ; \Omega)$ with the constant $1 / 2<\sigma \leq$ 1 , then we have

$$
\left\|\boldsymbol{v}-\Pi_{\mathcal{T}} \boldsymbol{v}\right\|_{\text {curl }} \lesssim h_{\mathcal{T}}^{\sigma}\|\boldsymbol{v}\|_{H^{\sigma}(\operatorname{curl} ; \Omega)} .
$$

We define the finite element space of $H_{0}^{1}(\Omega)$ corresponding to $\mathbb{V}(\mathcal{T})$ as

$$
\mathbb{S}(\mathcal{T}):=\left\{q \in H_{0}^{1}(\Omega),\left.q\right|_{K} \in \mathcal{P}_{j}, \forall K \in \mathcal{T}\right\}
$$

where

$$
j:= \begin{cases}l, & \mathbb{V}(\mathcal{T})=\mathbb{V}^{l, 1}(\mathcal{T}) \\ l+1, & \mathbb{V}(\mathcal{T})=\mathbb{V}^{l, 2}(\mathcal{T})\end{cases}
$$

It is important to notice that $\nabla \mathbb{S} \subset \mathbb{V}(\mathcal{T})$. We then introduce the orthogonal complement $\nabla \mathbb{S}$ in $\mathbb{V}(\mathcal{T})$ with respect to the $L^{2}$ inner product, $\mathbb{V}^{0}(\mathcal{T}):=\left\{\boldsymbol{v}_{\mathcal{T}} \in\right.$ $\left.\mathbb{V}(\mathcal{T}) \mid\left(\boldsymbol{v}_{\mathcal{T}}, \nabla q_{\mathcal{T}}\right)=0, \forall q_{\mathcal{T}} \in \mathbb{S}(\mathcal{T})\right\}$. We often say that the functions belonging to $\mathbb{V}^{0}(\mathcal{T})$ are the discrete divergence-free functions. The following lemma shows that the discrete divergence-free function can be well approximated by a continuous divergence-free function. The proof can be found, e.g., in [29] (Lemma 7.6).

Lemma 3.4. For any given $\boldsymbol{v}_{\mathcal{T}} \in \mathbb{V}^{0}(\mathcal{T})$, there exists a $\boldsymbol{v} \in \boldsymbol{H}_{0}(\mathbf{c u r l} ; \Omega)$ satisfying

$$
\begin{aligned}
& \nabla \times \boldsymbol{v}=\nabla \times \boldsymbol{v}_{\mathcal{T}}, \nabla \cdot \boldsymbol{v}=0, \text { and } \\
& \left\|\boldsymbol{v}-\boldsymbol{v}_{\mathcal{T}}\right\|_{0} \lesssim h_{\mathcal{T}}^{\sigma}\left\|\nabla \times \boldsymbol{v}_{\mathcal{T}}\right\|_{0} .
\end{aligned}
$$

with a constant $\sigma \in(1 / 2,1]$ depending only on $\Omega$. If $\Omega$ is smooth or convex, then $\sigma=1$.

Now we are in a position to prove Lemma 3.1

Proof of Lemma 3.1. We apply the discrete Helmholtz decompositions 29] to $\boldsymbol{u}_{\mathcal{T}_{*}}-$ $\boldsymbol{u}_{\mathcal{T}}$ : there exist $\boldsymbol{r}_{\mathcal{T}_{*}} \in \mathbb{V}^{0}\left(\mathcal{T}_{*}\right)$ and $p_{\mathcal{T}_{*}} \in \mathbb{S}\left(\mathcal{T}_{*}\right)$, such that

$$
\boldsymbol{u}_{\mathcal{T}_{*}}-\boldsymbol{u}_{\mathcal{T}}=\boldsymbol{r}_{\mathcal{T}_{*}}+\nabla p_{\mathcal{T}_{*}},
$$

which yields

$$
\nabla \times \boldsymbol{r}_{\mathcal{T}_{*}}=\nabla \times\left(\boldsymbol{u}_{\mathcal{T}_{*}}-\boldsymbol{u}_{\mathcal{T}}\right) .
$$

Note that the Galerkin orthogonality $\hat{a}\left(\boldsymbol{u}-\boldsymbol{u}_{\mathcal{T}_{*}}, \boldsymbol{v}_{*}\right)=0$ holds for any $\boldsymbol{v}_{\mathcal{T}_{*}} \in$ $\mathbb{V}\left(\mathcal{T}_{*}\right)$; in particular, by choosing $\boldsymbol{v}_{\mathcal{T}_{*}}=\nabla p_{\mathcal{T}_{*}}$, we obtain the $L^{2}$ orthogonality,

$$
\left(\boldsymbol{u}-\boldsymbol{u}_{\mathcal{T}_{*}}, \nabla p_{\mathcal{T}_{*}}\right)=0
$$

Using the definition of bilinear form $N(\cdot, \cdot)$, (3.7), (3.9), and the Cauchy-Schwarz inequality, we have

$$
\begin{aligned}
N\left(\boldsymbol{u}-\boldsymbol{u}_{\mathcal{T}_{*}}, \boldsymbol{u}_{\mathcal{T}_{*}}-\boldsymbol{u}_{\mathcal{T}}\right) & =-\left(\omega^{2}+1\right)\left(\boldsymbol{u}-\boldsymbol{u}_{\mathcal{T}_{*}}, \boldsymbol{u}_{\mathcal{T}_{*}}-\boldsymbol{u}_{\mathcal{T}}\right) \\
& =-\left(\omega^{2}+1\right)\left(\boldsymbol{u}-\boldsymbol{u}_{\mathcal{T}_{*}}, \boldsymbol{r}_{\mathcal{T}_{*}}+\nabla p_{\mathcal{T}_{*}}\right) \\
& \lesssim\left\|\boldsymbol{u}-\boldsymbol{u}_{\mathcal{T}_{*}}\right\|_{\text {curl }}\left\|\boldsymbol{r}_{\mathcal{T}_{*}}\right\|_{0} .
\end{aligned}
$$

For $\boldsymbol{r}_{\mathcal{T}_{*}}$ in (3.7), using Lemma 3.4 there exists $\boldsymbol{r} \in \boldsymbol{H}_{0}(\mathbf{c u r l} ; \Omega)$ which satisfies

$$
\nabla \times \boldsymbol{r}=\nabla \times \boldsymbol{r}_{\mathcal{T}_{*}}, \nabla \cdot \boldsymbol{r}=0
$$


and

$$
\left\|\boldsymbol{r}-\boldsymbol{r}_{\mathcal{T}_{*}}\right\|_{0} \lesssim h_{\mathcal{T}_{*}}^{\sigma}\left\|\nabla \times \boldsymbol{r}_{\mathcal{T}_{*}}\right\|_{0}
$$

Using (3.8) in (3.12), we have

$$
\left\|\boldsymbol{r}-\boldsymbol{r}_{\mathcal{T}_{*}}\right\|_{0} \lesssim h_{\mathcal{T}_{*}}^{\sigma}\left\|\nabla \times\left(\boldsymbol{u}_{\mathcal{T}_{*}}-\boldsymbol{u}_{\mathcal{T}}\right)\right\|_{0}
$$

Next, we use a duality argument to obtain the $L^{2}$ estimate of $\boldsymbol{r}$. Let $\boldsymbol{\Psi} \in$ $\boldsymbol{H}_{0}(\mathbf{c u r l} ; \Omega)$ be the solution to the following variational problem:

$$
\hat{a}(\boldsymbol{v}, \Psi)=(\boldsymbol{r}, \boldsymbol{v}), \text { for all } \boldsymbol{v} \in \boldsymbol{H}_{0}(\operatorname{curl} ; \Omega) .
$$

Noting that $\nabla \cdot \boldsymbol{r}=0$, and taking $\boldsymbol{v}=\nabla q$ with some $q \in H_{0}^{1}(\Omega)$ in (3.14), we have

$$
\omega^{2}(\nabla q, \boldsymbol{\Psi})=0 .
$$

Furthermore, we have the following regularity result (see [30]): for some constant $\sigma \in(1 / 2,1]$

$$
\|\Psi\|_{H^{\sigma}(\operatorname{curl} ; \Omega)} \lesssim\|\boldsymbol{r}\|_{0} .
$$

Combining (3.8) with (3.11), we have

$$
\nabla \times\left(\boldsymbol{r}-\left(\boldsymbol{u}_{\mathcal{T}_{*}}-\boldsymbol{u}_{\mathcal{T}}\right)\right)=0 .
$$

Noting that $\boldsymbol{r}-\left(\boldsymbol{u}_{\mathcal{T}_{*}}-\boldsymbol{u}_{\mathcal{T}}\right) \in \boldsymbol{H}_{0}(\mathbf{c u r l} ; \Omega)$ and using (3.17), then from the exact sequence property, there exists $p \in H_{0}^{1}(\Omega)$, such that

$$
\boldsymbol{r}-\left(\boldsymbol{u}_{\mathcal{T}_{*}}-\boldsymbol{u}_{\mathcal{T}}\right)=\nabla p
$$

Using (3.18) and (3.15), we have

$$
\hat{a}\left(\boldsymbol{r}-\left(\boldsymbol{u}_{\mathcal{T}_{*}}-\boldsymbol{u}_{\mathcal{T}}\right), \boldsymbol{\Psi}\right)=\hat{a}(\nabla p, \boldsymbol{\Psi})=-\omega^{2}(\nabla p, \boldsymbol{\Psi})=0 .
$$

Now, let $\boldsymbol{v}=\boldsymbol{r}$ in (3.14), then using (3.19), Galerkin orthogonality, the CauchySchwarz inequality, (3.16), and (3.5), we have

$$
\begin{aligned}
\|\boldsymbol{r}\|_{0}^{2} & =\hat{a}(\boldsymbol{r}, \boldsymbol{\Psi})=\hat{a}\left(\boldsymbol{r}-\left(\boldsymbol{u}_{\mathcal{T}_{*}}-\boldsymbol{u}_{\mathcal{T}}\right), \boldsymbol{\Psi}\right)+\hat{a}\left(\boldsymbol{u}_{\mathcal{T}_{*}}-\boldsymbol{u}_{\mathcal{T}}, \boldsymbol{\Psi}\right) \\
& =\hat{a}\left(\boldsymbol{u}_{\mathcal{T}_{*}}-\boldsymbol{u}_{\mathcal{T}}, \boldsymbol{\Psi}\right)=\hat{a}\left(\boldsymbol{u}_{\mathcal{T}_{*}}-\boldsymbol{u}_{\mathcal{T}}, \boldsymbol{\Psi}-\Pi_{\mathcal{T}} \boldsymbol{\Psi}\right) \\
& \lesssim\left\|\boldsymbol{u}_{\mathcal{T}_{*}}-\boldsymbol{u}_{\mathcal{T}}\right\|_{\text {curl }}\left\|\boldsymbol{\Psi}-\Pi_{\mathcal{T}} \boldsymbol{\Psi}\right\|_{\text {curl }} \\
& \lesssim h_{\mathcal{T}}^{\sigma}\left\|\boldsymbol{u}_{\mathcal{T}_{*}}-\boldsymbol{u}_{\mathcal{T}}\right\|_{\text {curl }}\|\boldsymbol{r}\|_{0} .
\end{aligned}
$$

Thus, we have proved that

$$
\|\boldsymbol{r}\|_{0} \lesssim h_{\mathcal{T}}^{\sigma}\left\|\boldsymbol{u}_{\mathcal{T}_{*}}-\boldsymbol{u}_{\mathcal{T}}\right\|_{\text {curl }}
$$

Using the triangle inequality, (3.13) and (3.20), and noting that $h_{\mathcal{T}_{*}} \leq h_{\mathcal{T}}$, we have

$$
\left\|\boldsymbol{r}_{*}\right\|_{0} \leq\left\|\boldsymbol{r}-\boldsymbol{r}_{\mathcal{T}_{*}}\right\|_{0}+\|\boldsymbol{r}\|_{0} \leq C h_{\mathcal{T}}^{\sigma}\left\|\boldsymbol{u}_{\mathcal{T}_{*}}-\boldsymbol{u}_{\mathcal{T}}\right\|_{\text {curl }} .
$$

Substituting (3.21) into (3.10), and choosing $h\left(\delta_{0}\right)$ sufficiently small such that $C h\left(\delta_{0}\right)^{\sigma} \leq \delta_{0}$, then for all $h_{\mathcal{T}}<h\left(\delta_{0}\right)$, we obtain the desired estimate (3.1). 
3.3. Convergence. We first recall three main ingredients to establish the convergence of AEFEM: a quasi-orthogonality, an upper bound of the a posteriori error estimator, and the reduction of the error estimator.

Recalling the quasi-orthogonality: for any given $\delta_{0}>0$, when the initial grid is sufficiently fine, for $\mathcal{T} \leq \mathcal{T} *$, we have

$$
\left\|\boldsymbol{u}-\boldsymbol{u}_{\mathcal{T}_{*}}\right\|_{\text {curl }}^{2} \leq \frac{1}{1-\delta_{0}}\left\|\boldsymbol{u}-\boldsymbol{u}_{\mathcal{T}}\right\|_{\text {curl }}^{2}-\left\|\boldsymbol{u}_{\mathcal{T}_{*}}-\boldsymbol{u}_{\mathcal{T}}\right\|_{\text {curl }}^{2}
$$

The following a posteriori upper bound can be obtained by adapting the results in Schöberl 38 to the indefinite case easily.

Lemma 3.5. Let $\boldsymbol{u} \in \boldsymbol{H}_{0}(\mathbf{c u r l} ; \Omega)$ be the solution of (2.1), $\mathcal{T} \in \mathscr{C}\left(\mathcal{T}_{0}\right)$, and let $\boldsymbol{u}_{\mathcal{T}} \in \mathbb{V}(\mathcal{T})$ be the discrete solutions of $(2.3)$. Then there exists a constant $C_{1}>0$ depending only on the shape regularity of $\mathcal{T}$ and the wave number $\omega$, such that

$$
\left\|\boldsymbol{u}-\boldsymbol{u}_{\mathcal{T}}\right\|_{\text {curl }}^{2} \leq C_{1} \eta^{2}\left(\boldsymbol{u}_{\mathcal{T}}, \mathcal{T}\right)
$$

The reduction of the error estimator between two consecutive triangulations can be proved using similar arguments for the elliptic case [8] and also skipped here.

Lemma 3.6. There exists $\beta \in(0,1)$ depending only on the shape regularity of $\mathcal{T}_{k}$ and the parameter $\theta$ used in the marking strategy, such that

$$
\eta^{2}\left(\boldsymbol{u}_{k+1}, \mathcal{T}_{k+1}\right) \leq \beta \eta^{2}\left(\boldsymbol{u}_{k}, \mathcal{T}_{k}\right)+C_{\beta}\left\|\boldsymbol{u}_{k+1}-\boldsymbol{u}_{k}\right\|_{\text {curl }}^{2}
$$

where the constant $C_{\beta}>1$ depends only on $\beta$.

Now we prove the contraction of the summation of error and a scaled error indicate. Similar to elliptic equations, each term of the summation may not strictly decay. The corresponding discussion for elliptic equations can be found in [32.

Theorem 3.7. Assume the initial mesh size $h_{0}$ is fine enough, and for a given $\theta \in(0,1)$, let $\left\{\mathcal{T}_{k}, \boldsymbol{u}_{k}\right\}_{k \geq 0}$ be a sequence of meshes, and finite element solutions produced by the AEFEM. Then there exists constants $\rho \in(0,1)$, and $\delta \in(0,1)$, depending only on $\theta$ and the shape regularity of $\mathcal{T}_{0}$, such that

$$
\left\|\boldsymbol{u}-\boldsymbol{u}_{k+1}\right\|_{\text {curl }}^{2}+\rho \eta^{2}\left(\boldsymbol{u}_{k+1}, \mathcal{T}_{k+1}\right) \leq \delta\left(\left\|\boldsymbol{u}-\boldsymbol{u}_{k}\right\|_{\text {curl }}^{2}+\rho \eta^{2}\left(\boldsymbol{u}_{k}, \mathcal{T}_{k}\right)\right)
$$

Proof. We fix a $\beta$ in Lemma 3.6] and let $\rho:=C_{\beta}^{-1} \in(0,1)$. We then choose $\delta_{0}$ satisfying

$$
\delta_{0}<1-\left(1+C_{1}^{-1} \rho(1-\beta)\right)^{-1}
$$

and let

$$
\delta=\frac{\left(1-\delta_{0}\right)^{-1} C_{1}+\rho \beta}{C_{1}+\rho} .
$$

By the choice of $\delta_{0}$, we have $\delta_{0}, \delta \in(0,1)$ and $\delta<\left(1-\delta_{0}\right)^{-1}$. 
By adding $\rho \eta^{2}\left(\boldsymbol{u}_{k+1}, \mathcal{T}_{k+1}\right)$ to both sides of (3.22), then splitting $\left\|\boldsymbol{u}-\boldsymbol{u}_{k}\right\|_{\text {curl }}^{2}$ and applying Lemma 3.6 to cancel $\left\|\boldsymbol{u}_{k+1}-\boldsymbol{u}_{k}\right\|_{\text {curl }}^{2}$, we obtain

$$
\begin{aligned}
\| \boldsymbol{u} & -\boldsymbol{u}_{k+1} \|_{\text {curl }}^{2}+\rho \eta^{2}\left(\boldsymbol{u}_{k+1}, \mathcal{T}_{k+1}\right) \\
& \leq \frac{1}{1-\delta_{0}}\left\|\boldsymbol{u}-\boldsymbol{u}_{k}\right\|_{\text {curl }}^{2}-\left\|\boldsymbol{u}_{k+1}-\boldsymbol{u}_{k}\right\|_{\text {curl }}^{2}+\rho \eta^{2}\left(\boldsymbol{u}_{k+1}, \mathcal{T}_{k+1}\right) \\
& \leq \delta\left\|\boldsymbol{u}-\boldsymbol{u}_{k}\right\|_{\text {curl }}^{2}+\left(\frac{1}{1-\delta_{0}}-\delta\right)\left\|\boldsymbol{u}-\boldsymbol{u}_{k}\right\|_{\text {curl }}^{2}+\rho \beta \eta^{2}\left(\boldsymbol{u}_{k}, \mathcal{T}_{k}\right) \\
& \leq \delta\left(\left\|\boldsymbol{u}-\boldsymbol{u}_{k}\right\|_{\text {curl }}^{2}+\frac{\left[\left(1-\delta_{0}\right)^{-1}-\delta\right] C_{1}+\rho \beta}{\delta} \eta^{2}\left(\boldsymbol{u}_{k}, \mathcal{T}_{k}\right)\right)
\end{aligned}
$$

In the last step, we apply the upper bound (cf. Lemma 3.5) to $\left\|\boldsymbol{u}-\boldsymbol{u}_{k}\right\|_{\text {curl }}^{2}$.

Noting that by the definition (3.24),

$$
\rho=\frac{\left[\left(1-\delta_{0}\right)^{-1}-\delta\right] C_{1}+\rho \beta}{\delta},
$$

we then obtain

$$
\left\|\boldsymbol{u}-\boldsymbol{u}_{k+1}\right\|_{\text {curl }}^{2}+\rho \eta^{2}\left(\boldsymbol{u}_{k+1}, \mathcal{T}_{k+1}\right) \leq \delta\left(\left\|\boldsymbol{u}-\boldsymbol{u}_{k}\right\|_{\text {curl }}^{2}+\rho \eta^{2}\left(\boldsymbol{u}_{k}, \mathcal{T}_{k}\right)\right),
$$

which completes the proof.

By recursion, we get the geometric decay of the error plus the estimator.

Corollary 3.8. Under the hypotheses of Theorem 3.7, we have

$$
\left\|\boldsymbol{u}-\boldsymbol{u}_{k}\right\|_{\text {curl }}^{2}+\rho \eta^{2}\left(\boldsymbol{u}_{k}, \mathcal{T}_{k}\right) \leq \hat{C}_{0} \delta^{k},
$$

where the constant $\rho$ and $\delta$ are given in Theorem $\left[3.7\right.$, and $\hat{C}_{0}:=\left\|\boldsymbol{u}-\boldsymbol{u}_{0}\right\|_{\text {curl }}^{2}+$ $\rho \eta^{2}\left(\boldsymbol{u}_{0}, \mathcal{T}_{0}\right)$. Thus the algorithm AEFEM will terminate in finite steps.

\section{A STABLE AND LOCAL PROJECTION OPERATOR}

To prepare for the the quasi-optimality analysis of our AEFEM in this section, we shall construct a stable and local projection operator between two consecutive finite element spaces, which is the key to establish a localized upper bound. In our construction we shall use the following operators:

(1) the cut-off operator $\chi_{\mathcal{R}}: \mathbb{V}\left(\mathcal{T}_{*}\right) \rightarrow \mathbb{V}(\tilde{\mathcal{R}})$;

(2) the canonical edge interpolation $\Pi_{\mathcal{T}_{*}}^{\text {curl }}$ based on path integrals along edges;

(3) the Schöberl quasi-interpolation $\mathcal{S}_{\mathcal{T}}^{\text {curl }}: \mathbb{V}\left(\mathcal{T}_{*}\right) \rightarrow \mathbb{V}(\mathcal{T})$;

(4) the Scott-Zhang quasi-interpolation $\mathcal{Q}_{\mathcal{T}}: H_{0}^{1}(\Omega) \mapsto \mathbb{U}(\mathcal{T})$, where $\mathbb{U}(\mathcal{T}) \subset$ $H_{0}^{1}(\Omega)$ is the continuous and linear finite element space for mesh $\mathcal{T}$.

None of the above operators can achieve the locality (local projection) and stability simultaneously. For example, the canonical interpolation operator and ScottZhang operator can preserve the finite element function (and thus are local projection operators) but they can only apply to smoother functions and are not stable in $H$ (curl)-norm. Schöberl's quasi-interpolation is local and stable but cannot preserve finite element functions. There exist operators [37, 3, 11] which are stable and (global) projection but the locality is lost in the construction.

The idea of our construction is as follows. We decompose a function $\boldsymbol{v} \in \mathbb{V}\left(\mathcal{T}_{*}\right)$ into smooth parts (in $H^{1}$ ) and a non-smooth part (but of high frequency). For smooth parts, we apply Scott-Zhang quasi-interpolation, and for high frequency parts, we apply cut-off operator and Schöberl interpolation. The main result is summarized below. 
Theorem 4.1. For $\mathcal{T}, \mathcal{T}_{*} \in \mathscr{C}\left(\mathcal{T}_{0}\right)$ with $\mathcal{T} \leq \mathcal{T}_{*}$, let $\mathcal{R}=\mathcal{R}_{\mathcal{T} \rightarrow \mathcal{T}_{*}}=\{K \in$ $\mathcal{T}$, but $\left.K \notin \mathcal{T}_{*}\right\}$ be the set of refined elements from $\mathcal{T}$ to $\mathcal{T}_{*}, \tilde{\mathcal{R}}=\tilde{\mathcal{R}}_{\mathcal{T} \rightarrow \mathcal{T}_{*}}=\{K \in$ $\mathcal{T} \mid K \cap K^{\prime} \neq \varnothing$ for some $\left.K^{\prime} \in \mathcal{R}\right\}$. There exists a quasi-interpolation operator $\mathcal{I}_{\mathcal{T}}: \mathbb{V}\left(\mathcal{T}_{*}\right) \mapsto \mathbb{V}(\mathcal{T})$ such that, for $\boldsymbol{v} \in \mathbb{V}\left(\mathcal{T}_{*}\right)$ :

(1) $\mathcal{I}_{\mathcal{T}}$ is a local projection, i.e., $\left.\mathcal{I}_{\mathcal{T}} \boldsymbol{v}\right|_{\mathcal{T} \backslash \tilde{\mathcal{R}}}=\left.\boldsymbol{v}\right|_{\mathcal{T} \backslash \tilde{\mathcal{R}}}$.

(2) $\mathcal{I}_{\mathcal{T}}$ is stable in the $H$ (curl)-norm, i.e.,

$$
\left\|\mathcal{I}_{\mathcal{T}} \boldsymbol{v}\right\|_{\text {curl }} \lesssim\|\boldsymbol{v}\|_{\text {curl }} \text {. }
$$

4.1. Various interpolation operators. In the following, we introduce several existing operators along with their properties.

4.1.1. The cut-off operator. Let $\left\{\boldsymbol{\phi}_{i}^{*}\right\}$ be the basis functions for the space $\mathbb{V}\left(\mathcal{T}_{*}\right)$. Then for any given $\boldsymbol{v} \in \mathbb{V}\left(\mathcal{T}_{*}\right)$, we have

$$
\boldsymbol{v}=\sum_{i \in \mathcal{R}^{*}} \alpha_{i}^{*} \phi_{i}^{*}+\sum_{i \notin \mathcal{R}^{*}} \alpha_{i} \phi_{i}
$$

where $\mathcal{R}_{i}^{*}$ is the index set such that $\phi_{i}^{*} \notin \mathbb{V}(\mathcal{T})$, namely the set for the new bases added or changed by the refinement. We define

$$
\chi_{\mathcal{R}} \boldsymbol{v}=\sum_{i \in \mathcal{R}^{*}} \alpha_{i}^{*} \phi_{i}^{*} .
$$

That is, we simply cut off parts of the function values in the unrefined region $\mathcal{T} \backslash \tilde{\mathcal{R}}$, i.e., $\left.\chi_{\mathcal{R}} \boldsymbol{v}\right|_{\mathcal{T} \backslash \tilde{\mathcal{R}}}=0$ and $\left.\chi_{\mathcal{R}} \boldsymbol{v}\right|_{\mathcal{R}}=\boldsymbol{v}$. Obviously, $\boldsymbol{v}-\chi_{\mathcal{R}} \boldsymbol{v} \in \mathbb{V}(\mathcal{T})$.

Since the basis decomposition is $L^{2}$ stable [19], we get the stability of $\chi_{\mathcal{R}}$ in the $L^{2}$-norm

$$
\left\|\chi_{\mathcal{R}} \boldsymbol{v}\right\|_{0}^{2} \lesssim \sum_{i \in \mathcal{R}^{*}}\left\|\boldsymbol{v}_{i}^{*}\right\|_{0}^{2} \leq\|\boldsymbol{v}\|_{0}^{2} .
$$

However, $\chi_{\mathcal{R}}$ is not stable in $H($ curl)-norm due to the existence of the low frequency. As a simple example, we consider two hat basis functions in an interval with size $h$. Let $v=\phi_{1}+\phi_{2}=1$ and thus $v^{\prime}=0$. Suppose $\chi_{\mathcal{R}} v=\phi_{1}$. Then $\left(\chi_{\mathcal{R}} v\right)^{\prime}=1 / h$ and $\left\|\left(\chi_{\mathcal{R}} v\right)^{\prime}\right\|_{0}$ cannot be bounded by $\|v\|_{0}$.

The cut-off operator $\chi_{\mathcal{R}}$ will be stable restricted to high frequency. Let $h$ be the size function of the triangulation $\mathcal{T}_{*}$. A function $\tilde{\boldsymbol{v}} \in \mathbb{V}\left(\mathcal{T}_{*}\right)$ is of high frequency if $\left\|h^{-1} \tilde{\boldsymbol{v}}\right\|_{0} \lesssim\|\tilde{\boldsymbol{v}}\|_{\text {curl }}$. Then using the inverse inequality, the stability of $\chi_{\mathcal{R}}$ in the $L^{2}$-norm, and the definition of high frequency, we have

$$
\left\|\chi_{\mathcal{R}} \tilde{\boldsymbol{v}}\right\|_{\text {curl }} \lesssim\left\|h^{-1} \chi_{\mathcal{R}} \tilde{\boldsymbol{v}}\right\|_{0} \lesssim\left\|h^{-1} \tilde{\boldsymbol{v}}\right\|_{0} \lesssim\|\tilde{\boldsymbol{v}}\|_{\text {curl }} .
$$

4.1.2. Canonical edge interpolation. For a general conforming triangulation $\mathcal{T}$, let $\mathcal{E}(\mathcal{T})$ be the set of interior edges of the mesh $\mathcal{T}$. We can define the canonical edge interpolation $\Pi_{\mathcal{T}}^{\text {curl }} \boldsymbol{v} \in \mathbb{V}^{1,1}(\mathcal{T})$ for a smooth enough function $\boldsymbol{v}$ as

$$
\Pi_{\mathcal{T}}^{\text {curl }} \boldsymbol{v}=\sum_{e \in \mathcal{E}(\mathcal{T})}\left(\int_{e} \boldsymbol{v} \cdot \mathrm{d} \boldsymbol{s}\right) \phi_{e},
$$

where $\phi_{e}$ is the edge element basis function associated with the edge $e$.

The canonical edge interpolation $\Pi_{\mathcal{T}}^{\text {curl }}$ has several nice properties: it is a locally defined projection and satisfies the commuting diagram property [19, 29]. The main constraint is that it is not stable in $H($ curl)-norm. Indeed, it is even not well defined for $H$ (curl) functions. 
It can, however, be shown that $\Pi_{\mathcal{T}}^{\text {curl }}$ is well defined and stable in $H($ curl)-norm restricted to the continuous and piecewise linear finite element space $\mathbb{U}^{3}(\mathcal{T}$ ) (see Section 3.6 of [19] or Theorem 5.41 [29]):

$$
\left\|\Pi_{\mathcal{T}}^{\text {curl }} \boldsymbol{q}\right\|_{\text {curl }} \lesssim\|\boldsymbol{q}\|_{\text {curl }}, \quad \text { for all } \boldsymbol{q} \in \mathbb{U}^{3}(\mathcal{T}) .
$$

4.1.3. Schöberl quasi-interpolation. A sequence of quasi-interpolations $\mathcal{S}_{\mathcal{T}}^{D}(D=$ grad, curl, div) are constructed in [36] with the following nice properties:

(1) $\mathcal{S}_{\mathcal{T}}^{D}$ is well defined for $L^{2}$ functions and stable in $L^{2}$-norm.

(2) It commutes with differential operators: $\operatorname{curl} \mathcal{S}_{\mathcal{T}}^{\text {curl }}=\mathcal{S}_{\mathcal{T}}^{\text {div }}$ curl.

(3) It is locally defined. Their degrees of freedom are only associated with the local enlarged patch of edges or faces of the mesh.

Note that the properties (1) and (2) imply $\mathcal{S}_{\mathcal{T}}^{\text {curl }}$ is also stable in $H$ (curl)-norm by using the following argument:

$$
\left\|\operatorname{curl} \mathcal{S}_{\mathcal{T}}^{\text {curl }} \boldsymbol{v}\right\|_{0}=\left\|\mathcal{S}_{\mathcal{T}}^{\text {div }} \operatorname{curl} \boldsymbol{v}\right\|_{0} \lesssim\|\operatorname{curl} \boldsymbol{v}\|_{0}
$$

The main drawback of $\mathcal{S}_{\mathcal{T}}^{\text {curl }}$ is that it is not a projection, i.e., $\left(\mathcal{S}_{\mathcal{T}}^{\text {curl }}\right)^{2} \neq \mathcal{S}_{\mathcal{T}}^{\text {curl }}$, although it is locally defined. A remedy to get a stable projection is to compose it with a right inverse; see [37, 3, 11]. But the right inverse is in general a global operator and thus cannot preserve the function in the non-refined region.

4.1.4. Scott-Zhang quasi-interpolation. For $H^{1}$ functions, we often use the ScottZhang quasi-interpolation 39.

By the definition, $\mathcal{Q}_{\mathcal{T}}$ is a local projection and stable in $H^{1}$-norm (see [39]):

$$
\left\|\mathcal{Q}_{\mathcal{T}} p\right\|_{1} \lesssim\|p\|_{1}, \quad \text { for all } p \in H_{0}^{1}(\Omega) \text {. }
$$

Similar operators such as $\mathcal{Q}_{\mathbb{S}(\mathcal{T})}$ can also be defined for high order spaces $\mathbb{S}(\mathcal{T})$, which are given by (3.6), and still be a local projection and stable in $H^{1}$-norm. More details on constructions can be found in 39].

For vector fields, we apply the Scott-Zhang quasi-interpolation to their components, separately, and still use the same symbol $\mathcal{Q}_{\mathcal{T}}$.

4.2. Discrete regular decomposition. Let us first assume that the continuous and linear finite element space $\mathbb{U}\left(\mathcal{T}_{*}\right)$ is a subspace of the edge element space $\mathbb{V}\left(\mathcal{T}_{*}\right)$ (which holds except for the lowest-order element of the first family $\mathbb{V}^{1,1}\left(\mathcal{T}_{*}\right)$ ). Then, for any $\boldsymbol{v} \in \mathbb{V}\left(\mathcal{T}_{*}\right)$, we have a discrete regular decomposition [20, 45],

$$
\boldsymbol{v}=\tilde{\boldsymbol{v}}+\boldsymbol{\phi}+\nabla p,
$$

where $\tilde{\boldsymbol{v}} \in \mathbb{V}\left(\mathcal{T}_{*}\right)$ is of high frequency and $\phi \in \mathbb{U}^{3}\left(\mathcal{T}_{*}\right) \subset H_{0}^{1}(\Omega)$ and $p \in \mathbb{S}(\mathcal{T}) \subset$ $H_{0}^{1}(\Omega)$; cf. (3.6) for the definition of $\mathbb{S}(\mathcal{T})$. The decomposition is stable in the sense that

$$
\left\|h^{-1} \tilde{\boldsymbol{v}}\right\|_{0}+\|\boldsymbol{\phi}\|_{1}+\|p\|_{1} \lesssim\|\boldsymbol{v}\|_{\text {curl }} .
$$

4.3. A stable and local projection operator. For any $v \in \mathbb{V}\left(\mathcal{T}_{*}\right)$, by the discrete regular decomposition (4.3), we have

$$
\boldsymbol{v}=\chi_{\mathcal{R}} \tilde{\boldsymbol{v}}+\left(\tilde{\boldsymbol{v}}-\chi_{\mathcal{R}} \tilde{\boldsymbol{v}}\right)+\boldsymbol{\phi}+\nabla p .
$$

We define $\mathcal{I}_{\mathcal{T}} \boldsymbol{v} \in \mathbb{V}$ by

$$
\mathcal{I}_{\mathcal{T}} \boldsymbol{v}=\mathcal{S}_{\mathcal{T}}^{\text {curl }} \chi_{\mathcal{R}} \tilde{\boldsymbol{v}}+\left(\tilde{\boldsymbol{v}}-\chi_{\mathcal{R}} \tilde{\boldsymbol{v}}\right)+\mathcal{Q}_{\mathcal{T}} \phi+\nabla \mathcal{Q}_{S(\mathcal{T})} p .
$$


That is, we apply Scott-Zhang interpolation to the smooth parts and the cut-off and Schöberl interpolation to the high frequency part. Here we use the fact that the linear finite element space $\mathbb{U}(\mathcal{T})$ is in $\mathbb{V}(\mathcal{T})$ and $\nabla \mathbb{S}(\mathcal{T}) \subset \mathbb{V}(\mathcal{T})$.

The difference is that

$$
\boldsymbol{v}-\mathcal{I}_{\mathcal{T}} \boldsymbol{v}=\left(I d-\mathcal{S}_{\mathcal{T}}^{\text {curl }}\right) \chi_{\mathcal{R}} \tilde{\boldsymbol{v}}+\left(I d-\mathcal{Q}_{\mathcal{T}}\right) \boldsymbol{\phi}+\nabla\left(I d-\mathcal{Q}_{S(\mathcal{T})}\right) p
$$

noting that the cut-off interpolation satisfies $\left.\chi_{\mathcal{R}} \tilde{\boldsymbol{v}}\right|_{\mathcal{T} \backslash \tilde{\mathcal{R}}}=0$. By choosing appropriate faces for each degree of freedom in the Scott-Zhang quasi-interpolation, we can enforce $\left.\left(I d-\mathcal{Q}_{\mathcal{T}}\right) \phi\right|_{\mathcal{T} \backslash \tilde{\mathcal{R}}}=0$ and $\left.\left(I d-\mathcal{Q}_{S(\mathcal{T})}\right) p\right|_{\mathcal{T} \backslash \tilde{\mathcal{R}}}=0$. Therefore $\boldsymbol{v}-\mathcal{I}_{\mathcal{T}} \boldsymbol{v}$ is vanished in $\mathcal{T} \backslash \tilde{\mathcal{R}}$.

Now we prove that $\mathcal{I}_{\mathcal{T}}$ is stable in $H($ curl)-norm or, equivalently,

$$
\left\|\boldsymbol{v}-\mathcal{I}_{\mathcal{T}} \boldsymbol{v}\right\|_{\text {curl }} \lesssim\|\boldsymbol{v}\|_{\text {curl }} .
$$

In view of (4.5), we divide our proof into three parts.

(1) For the first part, using the stability of $\mathcal{S}_{\mathcal{T}}^{\text {curl }}$ and the stability of $\chi_{\mathcal{R}}$ restricted to high frequency, we get

$\left\|\left(I d-\mathcal{S}_{\mathcal{T}}^{\text {curl }}\right) \chi_{\mathcal{R}} \tilde{\boldsymbol{v}}\right\|_{\text {curl }} \lesssim\left\|\chi_{\mathcal{R}} \tilde{\boldsymbol{v}}\right\|_{\text {curl }} \lesssim\|\tilde{\boldsymbol{v}}\|_{\text {curl }} \lesssim\left\|h^{-1} \boldsymbol{v}\right\| \lesssim\|\boldsymbol{v}\|_{\text {curl }}$.

(2) For the second part, using the stability of $\mathcal{Q}_{\mathcal{T}}(4.2)$ and the stability of the decomposition (4.4), we get

$$
\left\|\mathcal{Q}_{\mathcal{T}} \phi\right\|_{\text {curl }} \leq\left\|\mathcal{Q}_{\mathcal{T}} \phi\right\|_{1} \lesssim\|\phi\|_{1} \lesssim\|v\|_{\text {curl }}
$$

(3) For the third part, we only need to consider the $L^{2}$-norm, since $\operatorname{curl} \nabla \mathcal{Q}_{\mathcal{T}} p$ $=0$. Then, using (4.2) and (4.4) again, we get

$$
\left\|\nabla \mathcal{Q}_{S(\mathcal{T})} p\right\|_{0} \leq\left\|\mathcal{Q}_{S(\mathcal{T})} p\right\|_{1} \lesssim\|p\|_{1} \lesssim\|\boldsymbol{v}\|_{\text {curl }}
$$

4.4. Lowest order edge element space. For the lowest-order element of the first family $\mathbb{V}^{1,1}\left(\mathcal{T}_{*}\right)$, the discrete regular decomposition is of the form

$$
\boldsymbol{v}=\tilde{\boldsymbol{v}}+\Pi_{\mathcal{T}_{*}}^{\text {curl }} \phi+\nabla p,
$$

where $\boldsymbol{v} \in \mathbb{V}^{1,1}\left(\mathcal{T}_{*}\right), \tilde{\boldsymbol{v}} \in \mathbb{V}^{1,1}\left(\mathcal{T}_{*}\right), \phi \in \mathbb{U}^{3}\left(\mathcal{T}_{*}\right), p \in \mathbb{U}\left(\mathcal{T}_{*}\right), \Pi_{\mathcal{T}_{*}}^{\text {curl }}: \mathbb{U}^{3}\left(\mathcal{T}_{*}\right) \mapsto$ $\mathbb{V}^{1,1}\left(\mathcal{T}_{*}\right)$. The decomposition is stable in the sense that (4.4) holds.

As in the previous case, we rewrite $\boldsymbol{v}$ as

$$
\boldsymbol{v}=\chi_{\mathcal{R}} \tilde{\boldsymbol{v}}+\left(\tilde{\boldsymbol{v}}-\chi_{\mathcal{R}} \tilde{\boldsymbol{v}}\right)+\Pi_{\mathcal{T}_{*}}^{\text {curl }} \boldsymbol{\phi}+\nabla p,
$$

and define $\mathcal{I}_{\mathcal{T}} \boldsymbol{v} \in \mathbb{V}(\mathcal{T})$,

$$
\mathcal{I}_{\mathcal{T}} \boldsymbol{v}=\mathcal{S}_{\mathcal{T}}^{\text {curl }} \chi_{\mathcal{R}} \tilde{\boldsymbol{v}}+\left(\tilde{\boldsymbol{v}}-\chi_{\mathcal{R}} \tilde{\boldsymbol{v}}\right)+\Pi_{\mathcal{T}}^{\text {curl }} \mathcal{Q}_{\mathcal{T}} \phi+\nabla \mathcal{Q}_{\mathcal{T}} p
$$

The difference is

$$
\boldsymbol{v}-\mathcal{I}_{\mathcal{T}} \boldsymbol{v}=\left(I d-\mathcal{S}_{\mathcal{T}}^{\text {curl }}\right) \chi_{\mathcal{R}} \tilde{\boldsymbol{v}}+\left(\Pi_{\mathcal{T}_{*}}^{\text {curl }} \phi-\Pi_{\mathcal{T}}^{\text {curl }} \mathcal{Q}_{\mathcal{T}} \phi\right)+\nabla\left(I d-\mathcal{Q}_{\mathcal{T}}\right) p .
$$

The first and third components are dealt with similarly as before. For the difference of the middle one, we first verify $\left.\left(\Pi_{\mathcal{T}_{*}}^{\text {curl }} \phi-\Pi_{\mathcal{T}}^{\text {curl }} \mathcal{Q}_{\mathcal{T}} \phi\right)\right|_{\mathcal{T} \backslash \tilde{\mathcal{R}}}=0$. In fact, note that $\mathcal{T} \backslash \tilde{\mathcal{R}}$ is a subset of the non-refined region and $\mathcal{Q}_{\mathcal{T}}$ is a local projection, then we have $\mathcal{Q}_{\mathcal{T}} \phi=\phi$ in $\mathcal{T} \backslash \tilde{\mathcal{R}}$. Furthermore, for an edge in the non-refined region $\mathcal{T} \backslash \tilde{\mathcal{R}}$, the two vertices are also in the non-refined region. So the corresponding line integrals and the edge basis in $\mathcal{T}_{*}$ and $\mathcal{T}$ are the same and, consequently, $\left.\left(\Pi_{\mathcal{T}_{*}}^{\text {curl }} \phi-\Pi_{\mathcal{T}}^{\text {curl }} \mathcal{Q}_{\mathcal{T}} \phi\right)\right|_{\mathcal{T} \backslash \tilde{\mathcal{R}}}=0$. 
The stability follows from the triangle inequality, the stability of canonical edge interpolation (4.1), the stability of $\mathcal{Q}_{\mathcal{T}}$ (4.2), and the stability of the discrete decomposition (4.4):

$$
\begin{gathered}
\left\|\Pi_{\mathcal{T}_{*}}^{\text {curl }} \phi-\Pi_{\mathcal{T}}^{\text {curl }} \mathcal{Q}_{\mathcal{T}} \phi\right\|_{\text {curl }} \lesssim\|\phi\|_{\text {curl }}+\left\|\mathcal{Q}_{\mathcal{T}} \phi\right\|_{\text {curl }} \lesssim\|\phi\|_{1} \lesssim\|\boldsymbol{v}\|_{\text {curl }} . \\
\text { 5. QUASI-OPTIMAL CARDINALITY OF THE AEFEM }
\end{gathered}
$$

\section{Quasi-Optimal CARdinality of the AEFEM}

In this section, we shall present the quasi-optimal cardinality of the AEFEM in terms of degrees of freedom (DOF) by assuming certain restrictions on the initial triangulation $\mathcal{T}_{0}$ and the marking parameter $\theta$. The key is to establish a localized upper bound for the difference between two finite element approximations.

5.1. Lower bound. We only use the upper bound of the error indicator (see Lemma 3.5 ) in the proof of convergence; this alone ensures that the error indicator $\eta$ is reliable, and that any amplification $\eta$ will also lead to a convergent algorithm. The efficiency of the estimator $\eta$ is important to make the optimal complexity possible.

For any given $\mathcal{T} \in \mathscr{C}\left(\mathcal{T}_{0}\right)$ and arbitrary $K \in \mathcal{T}$, we define the oscillation of $\boldsymbol{v}_{\mathcal{T}} \in \mathbb{V}(\mathcal{T})$ to be

$$
\begin{aligned}
\operatorname{osc}_{\mathcal{T}}^{2}\left(\boldsymbol{v}_{\mathcal{T}}, K\right)= & h_{K}^{2}\left(\left\|\left(I d-Q_{h_{K}}\right) R_{1}\left(\boldsymbol{v}_{\mathcal{T}}\right)\right\|_{0 ; K}^{2}+\left\|\left(I d-Q_{h_{K}}\right) R_{2}\left(\boldsymbol{v}_{\mathcal{T}}\right)\right\|_{0 ; K}^{2}\right) \\
& +\sum_{f \in K \cap \mathcal{F}(\mathcal{T})} h_{f}\left(\left\|\left(I d-Q_{h_{K}}\right) J_{1}\left(\boldsymbol{v}_{\mathcal{T}}\right)\right\|_{0 ; f}^{2}+\left\|\left(I d-Q_{h_{K}}\right) J_{2}\left(\boldsymbol{v}_{\mathcal{T}}\right)\right\|_{0 ; f}^{2}\right),
\end{aligned}
$$

where $Q_{h_{K}}$ denotes the $L^{2}$ projections onto the set of piecewise $\left(\mathcal{P}_{1}\right)^{3}$ or $\mathcal{P}_{1}$ over $K \in \mathcal{T}$ or $f \in \mathcal{F}(\mathcal{T})$, depending on the context.

Similar to the error indicator, for any subset $\mathcal{M} \subseteq \mathcal{T}$, we define

$$
\operatorname{osc}_{\mathcal{T}}^{2}\left(\boldsymbol{v}_{\mathcal{T}}, \mathcal{M}\right)=\sum_{K \in \mathcal{M}} \operatorname{osc}_{\mathcal{T}}^{2}\left(\boldsymbol{v}_{\mathcal{T}}, K\right), \quad \text { for all } \boldsymbol{v}_{\mathcal{T}} \in \mathbb{V}(\mathcal{T})
$$

When $\mathcal{M}=\mathcal{T}$, we shall simplify the notation as $\operatorname{osc}\left(\boldsymbol{v}_{\mathcal{T}}, \mathcal{T}\right)$.

The following lemma presents a lower bound for the error indicator. This can be proved by standard bubble function techniques [42] and simplifications of (2.3) for some special functions; see Izsák and van der Vegt [23].

Lemma 5.1 (Thm. 2 of [23]). Let $\boldsymbol{u} \in \boldsymbol{H}_{0}(\mathbf{c u r l} ; \Omega)$ be the solution of (2.1), $\mathcal{T} \in \mathscr{C}\left(\mathcal{T}_{0}\right)$, and let $\boldsymbol{u}_{\mathcal{T}} \in \mathbb{V}(\mathcal{T})$ be the discrete solution of (2.3). Then there exists a constant $C_{2}>0$ depending only on the shape regularity of $\mathcal{T}$ and parameter $\omega$, such that

$$
C_{2} \eta^{2}\left(\boldsymbol{u}_{\mathcal{T}}, \mathcal{T}\right) \leq\left\|\boldsymbol{u}-\boldsymbol{u}_{\mathcal{T}}\right\|_{\text {curl }}^{2}+\operatorname{osc}^{2}\left(\boldsymbol{u}_{\mathcal{T}}, \mathcal{T}\right)
$$

5.2. Localized upper bound. Unlike the elliptic case, since the difference between the discrete solutions of two nested meshes only has the regularity of $\boldsymbol{H}(\mathbf{c u r l} ; \Omega)$ and has a large kernel, we need to treat the kernel of the curl-operator and its orthogonal complement separately. Therefore we need the following localized regular decomposition of the error developed by Schöberl.

Theorem 5.2 (Thm. 1 of [38]). The Schöberl quasi-interpolation $\Pi_{\mathcal{T}}^{S}: \boldsymbol{H}_{0}(\mathbf{c u r l} ; \Omega)$ $\rightarrow \mathbb{V}^{1,1}(\mathcal{T})$ satisfies the following properties: For every $\boldsymbol{v} \in \boldsymbol{H}_{0}(\mathbf{c u r l} ; \Omega)$ there exist $\varphi \in H_{0}^{1}(\Omega)$ and $\boldsymbol{z} \in\left(H_{0}^{1}(\Omega)\right)^{3}$ such that

$$
\boldsymbol{v}-\Pi_{\mathcal{T}}^{S} \boldsymbol{v}=\nabla \varphi+\boldsymbol{z}
$$


The decomposition satisfies

$$
\begin{aligned}
h_{K}\|\varphi\|_{0 ; K}+\|\nabla \varphi\|_{0 ; K} & \lesssim\|\boldsymbol{v}\|_{\Omega_{K}}, \\
h_{K}\|\boldsymbol{z}\|_{0 ; K}+\|\nabla \boldsymbol{z}\|_{0 ; K} & \lesssim\|\nabla \times \boldsymbol{v}\|_{\Omega_{K}},
\end{aligned}
$$

where the constants depend only on the shape of the elements in $\Omega_{K}:=\left\{K^{\prime} \in\right.$ $\left.\mathcal{T}, K^{\prime} \cap K \neq \varnothing\right\}$, but do not depend on the global shape of the domain $\Omega$ or the size of $\Omega_{K}$.

Direct application of Schöberl's local decomposition cannot lead to the localized upper bound since the decomposition (5.1), $\varphi$ and $z$ may not vanish in the nonrefined region. We shall use our stable and local projection constructed in the previous section first, and then apply Schöberl's local decomposition.

Theorem 5.3. For $\mathcal{T}, \mathcal{T}_{*} \in \mathscr{C}\left(\mathcal{T}_{0}\right)$ with $\mathcal{T} \leq \mathcal{T}_{*}$, let $\mathcal{R}=\mathcal{R}_{\mathcal{T} \rightarrow \mathcal{T}_{*}}=\{K \in$ $\mathcal{T}$, but $\left.K \notin \mathcal{T}_{*}\right\}$ be the set of refined elements from $\mathcal{T}$ to $\mathcal{T}_{*}, \tilde{\mathcal{R}}=\tilde{\mathcal{R}}_{\mathcal{T} \rightarrow \mathcal{T}_{*}}=$ $\left\{K \in \mathcal{T} \mid K \cap K^{\prime} \neq \varnothing\right.$ for some $\left.K^{\prime} \in \mathcal{R}\right\}$. Let $\boldsymbol{u}_{\mathcal{T}} \in \mathbb{V}(\mathcal{T})$ and $\boldsymbol{u}_{\mathcal{T}_{*}} \in \mathbb{V}\left(\mathcal{T}_{*}\right)$ be the discrete solutions of (2.3). Then there exists a constant $C_{3}>0$, depending only on $\omega$, and the domain $\Omega$, such that

$$
\left\|\boldsymbol{u}_{\mathcal{T}_{*}}-\boldsymbol{u}_{\mathcal{T}}\right\|_{\text {curl }}^{2} \leq C_{3} \eta_{\mathcal{T}}^{2}\left(\boldsymbol{u}_{\mathcal{T}}, \tilde{\mathcal{R}}\right)
$$

Proof. Making use of the discrete inf-sup condition and Galerkin orthogonality, we have

$$
\begin{aligned}
\left\|\boldsymbol{u}_{\mathcal{T}_{*}}-\boldsymbol{u}_{\mathcal{T}}\right\|_{\text {curl }} & \lesssim \sup _{\boldsymbol{w}_{\mathcal{T}_{*}} \in \mathbb{V}\left(\mathcal{T}_{*}\right)} \frac{\hat{a}\left(\boldsymbol{u}_{\mathcal{T}_{*}}-\boldsymbol{u}_{\mathcal{T}}, \boldsymbol{w}_{\mathcal{T}_{*}}\right)}{\left\|\boldsymbol{w}_{\mathcal{T}_{*}}\right\|_{\text {curl }}} \\
& =\sup _{\boldsymbol{w}_{\mathcal{T}_{*}} \in \mathbb{V}\left(\mathcal{T}_{*}\right)} \frac{\hat{a}\left(\boldsymbol{u}_{\mathcal{T}_{*}}-\boldsymbol{u}_{\mathcal{T}}, \boldsymbol{w}_{\mathcal{T}_{*}}-\mathcal{I}_{\mathcal{T}} \boldsymbol{w}_{\mathcal{T}_{*}}\right)}{\left\|\boldsymbol{w}_{\mathcal{T}_{*}}\right\|_{\text {curl }}},
\end{aligned}
$$

where $\mathcal{I}_{\mathcal{T}}$ is the stable and local projection operator constructed in the previous section.

Denoted by $\widetilde{\boldsymbol{v}_{\mathcal{T}_{*}}}:=\boldsymbol{w}_{\mathcal{T}_{*}}-\mathcal{I}_{\mathcal{T}} \boldsymbol{w}_{\mathcal{T}_{*}} \in \boldsymbol{H}_{0}(\operatorname{curl}, \Omega)$. By Theorem 5.2 there exists a $\boldsymbol{\Phi} \in \boldsymbol{H}_{0}^{1}(\Omega)$ and a $p \in H_{0}^{1}(\Omega)$, such that

$$
\widetilde{\boldsymbol{v}_{\mathcal{T}_{*}}}-\Pi_{\mathcal{T}}^{S} \widetilde{\boldsymbol{v}_{\mathcal{T}_{*}}}=\mathbf{\Phi}+\nabla p
$$

and

$$
h_{K}\|p\|_{0 ; K}+\|\nabla p\|_{0 ; K} \lesssim\left\|\widetilde{\boldsymbol{v}_{\mathcal{T}_{*}}}\right\|_{\Omega_{K}}, h_{K}\|\boldsymbol{\Phi}\|_{0 ; K}+\|\nabla \boldsymbol{\Phi}\|_{0 ; K} \lesssim\left\|\nabla \times \widetilde{\boldsymbol{v}_{\mathcal{T}_{*}}}\right\|_{\Omega_{K}},
$$

Note that $\widetilde{\boldsymbol{v}_{\mathcal{T}_{*}}}=0$ in $\mathcal{T} \backslash \tilde{\mathcal{R}}$ implies $p=\boldsymbol{\Phi}=0$ in $\mathcal{T} \backslash \tilde{\mathcal{R}}$ by (5.5). Consequently,

$$
\widetilde{\boldsymbol{v}_{\mathcal{T}_{*}}}-\Pi_{\mathcal{T}}^{S} \widetilde{\boldsymbol{v}_{\mathcal{T}_{*}}}=0 \text { in } \mathcal{T} \backslash \tilde{\mathcal{R}}
$$


Using the definition of $\widetilde{\boldsymbol{v}_{\mathcal{T}_{*}}}$, Galerkin orthogonality (5.6), (5.4), the Green's formula, we have

$$
\begin{aligned}
\hat{a}\left(\boldsymbol{u}_{\mathcal{T}_{*}}-\boldsymbol{u}_{\mathcal{T}}, \boldsymbol{w}_{\mathcal{T}_{*}}-\mathcal{I}_{\mathcal{T}} \boldsymbol{w}_{\mathcal{T}_{*}}\right) & \\
= & \hat{a}\left(\boldsymbol{u}_{\mathcal{T}_{*}}-\boldsymbol{u}_{\mathcal{T}}, \widetilde{\boldsymbol{v}_{\mathcal{T}_{*}}}-\Pi_{\mathcal{T}}^{S} \widetilde{\boldsymbol{v}_{\mathcal{T}_{*}}}\right) \\
= & \sum_{K \in \tilde{\mathcal{R}}} \hat{a}_{K}\left(\boldsymbol{u}_{\mathcal{T}_{*}}-\boldsymbol{u}_{\mathcal{T}}, \boldsymbol{\Phi}+\nabla p\right) \\
= & \sum_{K \in \tilde{\mathcal{R}}}\left((\boldsymbol{g}, \boldsymbol{\Phi}+\nabla p)_{K}-\left(\nabla \times \boldsymbol{u}_{\mathcal{T}}, \nabla \times \boldsymbol{\Phi}\right)_{K}+\omega^{2}\left(\boldsymbol{u}_{\mathcal{T}}, \boldsymbol{\Phi}+\nabla p\right)_{K}\right) \\
= & \sum_{K \in \tilde{\mathcal{R}}}\left(\left(R_{1}\left(\boldsymbol{u}_{\mathcal{T}}\right), \boldsymbol{\Phi}\right)_{K}-\left(R_{2}\left(\boldsymbol{u}_{\mathcal{T}}\right), p\right)_{K}\right) \\
& +\sum_{f \in \mathscr{F}(\tilde{\mathcal{R}})}\left(\left(J_{1}\left(\boldsymbol{u}_{\mathcal{T}}\right), \boldsymbol{\Phi}\right)_{f}+\left(J_{2}\left(\boldsymbol{u}_{\mathcal{T}}\right)_{, p}\right)_{f}\right) \\
\leq & \sum_{K \in \tilde{\mathcal{R}}}\left(\left\|R_{1}\left(\boldsymbol{u}_{\mathcal{T}}\right)\right\|_{0 ; K}\|\boldsymbol{\Phi}\|_{0 ; K}+\left\|R_{2}\left(\boldsymbol{u}_{\mathcal{T}}\right)\right\|_{0 ; K}\|p\|_{0 ; K}\right) \\
& +\sum_{f \in \mathscr{F}(\tilde{\mathcal{R}})}\left(\left\|J_{1}\left(\boldsymbol{u}_{\mathcal{T}}\right)\right\|_{0 ; f}\|\boldsymbol{\Phi}\|_{0 ; f}+\left\|J_{2}\left(\boldsymbol{u}_{\mathcal{T}}\right)\right\|_{0 ; f}\|p\|_{0 ; f}\right) \\
\lesssim & \eta_{\mathcal{T}}\left(\boldsymbol{u}_{\mathcal{T}}, \tilde{\mathcal{R}}\right)\left\|\boldsymbol{w}_{\mathcal{T}_{*}}\right\|_{\text {curl }} .
\end{aligned}
$$

In the last step, we used the trace inequality $h_{f}^{-1}\|\phi\|_{0 ; f}^{2} \lesssim h_{f}^{-2}\|\phi\|_{0 ; K}^{2}+\|\nabla \phi\|_{0 ; K}^{2}$, $h_{f} \lesssim h_{K}$, (5.5) and (2).

The desired estimate (5.2) is a direct consequence of (5.3) and (5.7).

5.3. Approximation class. We follow the framework recently developed by Cascón, Kreuzer, Nochetto, and Siebert 8 for the general symmetric elliptic problem in order to define an approximation class.

We first introduce the so-called total error

$$
E_{\mathcal{T}}=\left(\left\|\boldsymbol{u}-\boldsymbol{u}_{\mathcal{T}}\right\|_{\text {curl }}^{2}+\operatorname{\rho osc}^{2}\left(\boldsymbol{u}_{\mathcal{T}}, \mathcal{T}\right)\right)^{1 / 2} .
$$

Using the quasi-orthogonality, we can obtain a quasi-monotonicity: for $\mathcal{T} \leq \mathcal{T}_{*}$, we have

$$
E_{\mathcal{T}_{*}} \leq\left(1-\delta_{0}\right)^{-1} E_{\mathcal{T}}
$$

Now we will define an approximation class $\mathcal{A}_{s}$ by making use of the total error. Let $\mathscr{C}\left(\mathcal{T}_{0}\right)_{N} \subset \mathscr{C}\left(\mathcal{T}_{0}\right)$ be the set of all possible conforming triangulations generated from $\mathcal{T}_{0}$ with at most $N$ elements more than $\mathcal{T}_{0}$ :

$$
\mathscr{C}\left(\mathcal{T}_{0}\right)_{N}:=\left\{\mathcal{T} \in \mathscr{C}\left(\mathcal{T}_{0}\right) \mid \# \mathcal{T}-\# \mathcal{T}_{0} \leq N\right\}
$$

We define the nonlinear approximation class $\mathcal{A}_{s}$ to be

$$
\mathcal{A}_{s}:=\left\{\left.(\boldsymbol{u}, \boldsymbol{g})||(\boldsymbol{u}, \boldsymbol{g})\right|_{\mathcal{A}_{s}}:=\sup _{N \geq N_{0}}\left(N^{s} \epsilon_{N}\right)<\infty, \text { with } \epsilon_{N}:=\min _{\mathcal{T} \in \mathscr{C}\left(\mathcal{T}_{0}\right)_{N}} E_{\mathcal{T}}\right\} .
$$

The characterization of $\mathcal{A}_{s}$ is beyond the scope of this paper. The index $s$ characterizes the best possible approximation rate, which depends on the regularity of the solution and data. To apply our adaptive algorithm, we do not need to know the value of $s$ explicitly. 
5.4. Quasi-optimality. The following result is a consequence of the previous estimates and the fact that the AEFEM is a contraction with respect to the sum of the energy error plus the scaled error estimator. The proof is a straightforward modification using the following ingredients: quasi-orthogonality, localized upper bound, lower bound, and thus skipped here. Details can be found in 46.

Theorem 5.4 (Quasi-Optimality). Given a $\theta \in\left(0, \theta_{*}\right)$ with the constant $\theta_{*}=$ $\frac{\rho C_{2}}{\rho+C_{3}\left(1+\delta_{0}+\rho C_{5}\right)}<1$, let $\boldsymbol{u}$ be the solution of (2.1), and let $\left\{\boldsymbol{u}_{k}, \mathcal{T}_{k}\right\}_{k \geq 0}$ be the sequence of discrete solutions and meshes produced by the AEFEM. Then, if $(\boldsymbol{u}, \boldsymbol{g}) \in$ $\mathcal{A}_{s}$, the initial mesh size $h_{0}$ is sufficiently small and the bisection method satisfies the assumption (B1) and (B2), we have

$$
\left(\left\|\boldsymbol{u}-\boldsymbol{u}_{k}\right\|_{\text {curl }}^{2}+\rho \operatorname{osc}^{2}\left(\boldsymbol{u}_{k},, \mathcal{T}_{k}\right)\right)^{1 / 2} \lesssim|(\boldsymbol{u}, \boldsymbol{g})|_{s}\left(\# \mathcal{T}_{k}-\# \mathcal{T}_{0}\right)^{-s}
$$

\section{ACKNOWLEDGMENTS}

The authors would like to thank Professor Ricardo H. Nochetto from the University of Maryland for the insightful comments on the localized upper bound, and Doctors Yunrong Zhu from the University of California at San Diego and Xuehai Huang from Pennsylvania State University, for discussions and a careful reading of the paper.

\section{REFERENCES}

1. A. Alonso and A. Valli, An optimal domain decomposition preconditioner for low-frequency time-harmonic Maxwell equations, Math. Comp. 68 (1999), no. 226, 607-631. MR 1609607 (99i:78002)

2. D. Arnold, R. Falk, and R. Winther, Multigrid in $H($ div $)$ and $H($ curl $)$, Numer. Math. 85 (2000), no. 2, 197-217. MR1754719(2001d:65161)

3. D. Arnold, R. Falk, and R. Winther, Finite element exterior calculus, homological techniques, and applications, Acta Numer. 15 (2006), 1-155. MR2269741(2007j:58002)

4. R. Beck, P. Deufhard, R. Hiptmair, R. Hoppe, and B. Wohlmuth, Adaptive multilevel methods for edge element discretizations of Maxwell's equations, Surveys Math. Industry 8 (1999), no. 3-4, 271-312. MR.1737416 (2000i:65206)

5. R. Beck, R. Hiptmair, R. Hoppe, and B. Wohlmuth, Residual based a posteriori error estimators for eddy current computation, M2AN Math. Model. Numer. Anal. 34 (2000), no. 1, 159-182. MR1735971 (2000k:65203)

6. P. Binev, W. Dahmen, and R. DeVore, Adaptive finite element methods with convergence rates, Numer. Math. 97 (2004), no. 2, 219-268. MR2050077(2005d:65222)

7. C. Carstensen and R. Hoppe, Convergence analysis of an adaptive edge finite element method for the 2D eddy current equations, J. Numer. Math. 13 (2005), no. 1, 19-32. MR.2130149 (2006b:65164)

8. J. Cascon, C. Kreuzer, R. Nochetto, and K. Siebert, Quasi-optimal convergence rate for an adaptive finite element method, SIAM J. Numer. Anal. 46 (2008), no. 5, 2524-2550. MR2421046 (2009h:65174)

9. L. Chen, R. Nochetto, and J. Xu, Multilevel methods on graded bisection grids II: H(curl) and $H($ div $)$ systems, Preprint, 2008.

10. Z. Chen, L. Wang, and W. Zheng, An adaptive multilevel method for time-harmonic Maxwell equations with singularities, SIAM J. Sci. Comput. 29 (2007), no. 1, 118-138. MR.2285885 (2008e:65342)

11. S. Christiansen and R. Winther, Smoothed projections in finite element exterior calculus, Math. Comp. 77 (2008), no. 262, 813-829. MR2373181 (2009a:65310)

12. M. Costabel and M. Dauge, Singularities of electromagnetic fields in polyhedral domains, Arch. Rational Mech. Anal. 151 (2000), no. 3, 221-276. MR1753704(2002c:78005)

13. M. Costabel, M. Dauge, and S. Nicaise, Singularities of eddy current problems, M2AN Math. Model. Numer. Anal. 37 (2003), no. 5, 807-831. MR2020865(2005a:35265) 
14. D. Dauge and R. Stevenson, Sparse tensor product wavelet approximation of singular functions, SIAM J. Math. Anal. 42 (2010), no. 5, 2203-2228.

Preprint 09-23, Universite de Rennes 1, (2009).

15. W. Dörfler, A convergent adaptive algorithm for Poisson's equation, SIAM J. Numer. Anal. 33 (1996), no. 3, 1106-1124. MR1393904(97e:65139)

16. J. Gopalakrishnan and J. Pasciak, Overlapping Schwarz preconditioners for indefinite time harmonic Maxwell equations, Math. Comp. 72 (2003), no. 241, 1-15. MR1933811 (2003i:78020)

17. J. Gopalakrishnan, J. Pasciak, and L.F. Demkowicz, Analysis of a multigrid algorithm for time harmonic Maxwell equations, SIAM J. Numer. Anal. 42 (2004), no. 1, 90-108. MR2051058 (2005c:65115)

18. R. Hiptmair, Multigrid method for Maxwell's equations, SIAM J. Numer. Anal. 36 (1999), no. 1, 204-225. MR:1654571 (99j:65229)

19. R. Hiptmair, Finite elements in computational electromagnetism, Acta Numer. 11 (2002), 237-339. MR2009375 (2004k:78028)

20. R. Hiptmair and J. Xu, Nodal auxiliary spaces preconditions in $H($ curl) and $H($ div $)$ spaces, SIAM J. Numer. Anal. 45 (2007), no. 6, 2483-2509. MR2361899(2009g:65153)

21. R. Hiptmair and W. Zheng, Local multigrid in $\boldsymbol{H}(\boldsymbol{c u r l} ; \Omega)$, J. Comput. Math. 27 (2009), no. 5, 573-603. MR2536903 (2010h:65249)

22. R. Hoppe and J. Schöberl, Convergence of adaptive edge element methods for the $3 D$ eddy currents equations, J. Comput. Math. 27 (2009), no. 5, 657-676. MR2536907 (2010i:65269)

23. F. Izsak and J. Van Der Vegt, A reliable and efficient implicit a posteriori error estimate technique for the time harmonic Maxwell equations, Tech. report, Internal report, Department of Applied Mathematics, University of Twente, Netherlands, 2007, http://eprints. eemcs. utwente. nl/11443/, M2AN, submitted for publication, 2007.

24. I. Kossaczkỳ, A recursive approach to local mesh refinement in two and three dimensions, J. Comp. Appl. Math. 55 (1994), no. 3, 275-288. MR1329875 (95m:65207)

25. K. Mekchay and R. Nochetto, Convergence of adaptive finite element methods for general second order linear elliptic PDEs, SIAM J. Numer. Anal. 43 (2005), no. 5, 1803-1827. MR 2192319 (2006i:65201)

26. W. Mitchell, A comparison of adaptive refinement techniques for elliptic problems, ACM Trans. Math. Software 15 (1989), no. 4, 326-347. MR.1062496

27. P. Monk, A finite element method for approximating the time-harmonic Maxwell equations, Numer. Math. 63 (1992), no. 1, 243-261. MR.1182977(94b:65134)

28. P. Monk, A posteriori error indicators for Maxwell's equations, J. Comp. Appl. Math. 100 (1998), no. 2, 173-190. MR1659117(2000k:78020)

29. P. Monk, Finite element methods for Maxwell equations, Numerical Mathematics and Scientific Computation, Oxford University Press, Oxford, 2003. MR2059447 (2005d:65003)

30. P. Monk, A simple proof of convergence for an edge element discretization of Maxwell's equations, Computational electromagnetics, Lect. Notes Comput. Sci. Eng., 28, Springer, Berlin, Springer, 2003, pp. 127-141. MR1986135(2004i:78024)

31. P. Morin, R. Nochetto, and K. Siebert, Data oscillation and convergence of adaptive FEM, SIAM J. Numer. Anal. 38 (2000), no. 2, 466-488. MR1770058 (2001g:65157)

32. P. Morin, R. Nochetto, and K. Siebert, Convergence of adaptive finite element methods, SIAM Rev. 44 (2002), no. 4, 631-658. MR1980447

33. J. Nédélec, Mixed finite elements in $R^{3}$, Numer. Math. 35 (1980), no. 3, 315-341. MR592160 (81k:65125)

34. J. Nédélec, A new family of mixed finite elements in $R^{3}$, Numer. Math. 50 (1986), no. 1, 57-81. MR864305 (88e:65145)

35. R. H. Nochetto, K. G. Siebert, and A. Veeser, Theory of adaptive finite element methods: An introduction. In R.A. Devore and A. Kunoth, editors. Multiscale, Nonlinear and Adaptive Approximation, Springer, 2009. MR2648380(2011k:65164)

36. J. Schöberl, Commuting quasi-interpolation operators for mixed finite elements, 2nd European Conference on Computational Mechanics, 2001, pp. 854-855.

37. J. Schöberl, A multilevel decomposition result in $H$ (curl), Computing and Visualization in Science (2005), 41-52.

38. J. Schöberl, A posteriori error estimates for Maxwell equations, Math. Comp. 77 (2008), no. 262, 633-649. MR2373173(2008m:78017) 
39. L. Scott and S. Zhang, Finite element interpolation of nonsmooth functions satisfying boundary conditions, Math. Comp. 54 (1990), no. 190, 483-493. MR 1011446 (90j:65021)

40. R. Stevenson, Optimality of a standard adaptive finite element method, Found. Comput. Math. 7 (2007), no. 2, 245-269. MR.2324418 (2008i:65272)

41. R. Stevenson, The completion of locally refined simplicial partitions created by bisection, Math. Comp. 77 (2008), no. 261, 227-241. MR2353951 (2008j:65219)

42. R. Verfürth, A review of a posteriori error estimation and adaptive mesh-refinement techniques, Advances in Numerical Mathematics. Wiley-Teubner, Chichester-Stuttgart, 1996.

43. J. Xu, Iterative methods by space decomposition and subspace correction, SIAM Rev. 34 (1992), no. 4, 581-613. MR1193013 (93k:65029)

44. J. Xu, A new class of iterative methods for nonselfadjoint or indefinite problems, SIAM J. Numer. Anal. 29 (1992), no. 2, 303-319. MR.1154268 (92k:65063)

45. J. Xu, L. Chen, and R. Nochetto, Optimal multilevel methods for H(grad), H(curl) and H(div) systems on graded and unstructured grids, Multiscale, Nonlinear and Adaptive Approximation, Springer R. DeVore, A. Kunoth (Eds.), Springer, 2009. MR 2648382

46. L. Zhong, Fast algorithms of edge element discretizations and adaptive finite element methods for two classes of maxwell equations, Ph.D. thesis, Xiangtan University, 2009.

47. L. Zhong, S. Shu, J. Wang, and J. Xu, Two-grid methods and precondtioners for timeharmonic Maxwell equations, Numer. Linear Algebra Appl., 2011, accepted.

48. L. Zhong, S. Shu, G. Wittum, and J. Xu, Optimal error estimates of the nedelec edge elements for Time-harmonic Maxwell's equations, J. Comput. Math. 27 (2009), no. 5, 563-572. MR2536902 (2010e:65220)

School of Mathematical and Computational Sciences, Xiangtan University, Hunan 411105, CHINA

Current address: School of Mathematics Sciences, South China Normal University, Guangzhou 510631, China

E-mail address: zhong@scnu.edu.cn

Department of Mathematics, University of California, Irvine, California 92697

E-mail address: chenlong@math.uci.edu

School of Mathematical and Computational Sciences, Xiangtan University, Hunan 411105, CHINA

E-mail address: shushi@xtu.edu.cn

Simulation and Modeling, Goethe Center for Scientific Computing, Goethe-University, Kettenhofweg 139, 60325 Frankfurt am Main, Germany

E-mail address: wittum@gcsc.uni-frankfurt.de

Department of Mathematics, Pennsylvania State University, University Park, PennSYLVANIA 16802

E-mail address: xu@math.psu.edu 